This is a self-archived version of an original article. This version may differ from the original in pagination and typographic details. Author(s): $\begin{aligned} & \text { Schuh, M.; Heiße, F.; Eronen, T.; Ketter, J.; Köhler-Langes, F.; Rau, S.; Segal, T.; } \\ & \text { Quint, W.; Sturm, S.; Blaum, K. }\end{aligned}$

Title: Image charge shift in high-precision Penning traps

Year: 2019

Version: Published version

Copyright: @ 2019 American Physical Society

Rights: In Copyright

Rights url: http://rightsstatements.org/page//nC/1.0/?language=en

Please cite the original version:

Schuh, M., Heiße, F., Eronen, T., Ketter, J., Köhler-Langes, F., Rau, S., Segal, T., Quint, W., Sturm, S., \& Blaum, K. (2019). Image charge shift in high-precision Penning traps. Physical Review A, 100(2), Article 23411. https://doi.org/10.1103/PhysRevA.100.023411 


\title{
Image charge shift in high-precision Penning traps
}

\author{
M. Schuh,${ }^{1, *}{ }^{*}$ F. Heiße,${ }^{1,2, \dagger}$ T. Eronen, ${ }^{3}$ J. Ketter, ${ }^{1,+}$ F. Köhler-Langes,,${ }^{1}$ S. Rau,,${ }^{1}$ T. Segal, ${ }^{1}$ \\ W. Quint, ${ }^{2}$ S. Sturm, ${ }^{1}$ and K. Blaum ${ }^{1}$ \\ ${ }^{1}$ Max-Planck-Institut für Kernphysik, Saupfercheckweg 1, 69117 Heidelberg, Germany \\ ${ }^{2}$ GSI Helmholtzzentrum für Schwerionenforschung GmbH, Planckstraße 1, 64291 Darmstadt, Germany \\ ${ }^{3}$ University of Jyväskylä, P.O. Box 35 (YFL), FI-40014 University of Jyväskylä, Jyväskylä, Finland
}

(Received 8 October 2018; revised manuscript received 28 March 2019; published 16 August 2019)

\begin{abstract}
An ion in a Penning trap induces image charges on the surfaces of the trap electrodes. These induced image charges are used to detect the ion's motional frequencies, but they also create an additional electric field, which shifts the free-space cyclotron frequency typically at a relative level of several $10^{-11}$. In various high-precision Penning-trap experiments, systematics and their uncertainties are dominated by this so-called image charge shift (ICS). The ICS is investigated in this work by a finite-element simulation and by a dedicated measurement technique. Theoretical and experimental results are in excellent agreement. The measurement is using singly stored ions alternately measured in the same Penning trap. For the determination of the ion's magnetron frequency with relative precision of better than 10 parts per billion, a Ramsey-like technique has been developed. In addition, numerical calculations are carried out for other Penning traps and agree with older ICS measurements.
\end{abstract}

DOI: 10.1103/PhysRevA.100.023411

\section{INTRODUCTION}

Single ions trapped in cryogenic Penning traps have enabled a multitude of high-precision experiments in mass spectrometry [1-4], stringent tests of QED in strong fields [5-7], several tests of the charge, parity, and time (CPT) reversal symmetry theorem [8,9] and special relativity [10], as well as high-precision measurements of fundamental constants [11]. With the exception of mass doublets, by comparing two very similar masses [1,3], the predominant systematic shift of all these measurements is the image charge shift (ICS). Here, the motion of the ion in the center of the Penning trap is slightly modified by the induced image charges on the trap electrodes. For instance, the ICS of the high-precision measurement of the atomic mass of the electron [11] via the bound-state $g$ factor is a factor of 130 larger than the absolute sum of all other systematic shifts and $95 \%$ of the total systematic uncertainty is given by the uncertainty of the ICS. In contrast to other systematics, the ICS cannot be reduced by lower temperature of the ion or an improved detection method. The only way to reduce this shift is the increase of the size of the Penning-trap electrodes as the ICS scales inversely with the trap radius cubed; see Sec. III. However, the induced image current detection signal decreases with the trap radius, which constrains a simple enlargement of the trap. For this reason, a precise measurement or calculation of the ICS is of great importance for all state-of-the-art high-precision Penning-trap experiments with ions.

\footnotetext{
*marc.schuh@mpi-hd.mpg.de

†This manuscript comprises parts of the Ph.D. theses of M. Schuh and F. Heiße.

${ }^{\ddagger}$ Present address: GSI Helmholtzzentrum für Schwerionenforschung GmbH, Planckstraße 1, 64291 Darmstadt, Germany.
}

So far, the only experimental approach to study the ICS is the measurement of its linear scaling with the number of trapped ions $[12,13]$. There, the ICS has been measured with a relative precision of $4 \%$. On the theoretical side, semianalytical descriptions of the ICS could only be formulated for simplified trap geometries until now $[14,15]$.

After a short introduction of Penning-trap basics in Sec. II and the general concept of the ICS in Sec. III, this article will present an experimental approach for the determination of the ICS, which aims for relative uncertainties of a few percent, in Sec. IV. Here, the ICS is determined experimentally with two single trapped ions. In this way, hardto-control ion-ion interaction, which occurs in the former measurement approaches [12], is completely avoided. This technique is based on a 1000 -fold improved determination of the magnetron frequency, which requires a Ramsey-like measurement technique [16]. In Sec. V, we introduce an improved simulation of the ICS based on the finite-element method (FEM). The simulation is able to predict the frequency shift correctly for the measurement and is applicable to all Penning-trap experiments. With a relative precision below $1 \%$, these ICS calculations are not any longer limited by the numerical precision of the simulation but by the machining tolerances of the trap electrodes. In Sec. VI, one can find a short introduction of the simulated Penning-trap setups. Finally, in Sec. VII, the experimental and theoretical results are summarized and compared to earlier measurements and calculations for various Penning-trap experiments.

\section{PENNING-TRAP BASICS}

In an ideal Penning trap, a homogeneous magnetic field $\boldsymbol{B}_{0}=B_{0} \boldsymbol{e}_{z}$ is combined with an electrostatic quadrupole potential to spatially confine an ion [17]. The electrostatic 


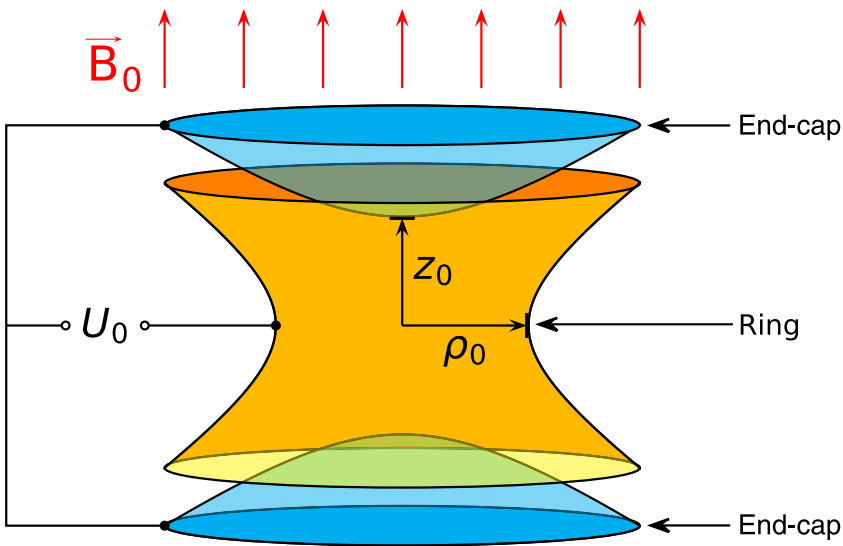

FIG. 1. A hyperbolic Penning trap consisting of a ring and two end-cap electrodes, with the characteristic trap parameters $z_{0}$ and $\rho_{0}$. The electric quadrupole potential is created by applying the voltage $U_{0}$ between the ring and the end caps. This electric potential is superimposed with a homogeneous magnetic field $B_{0}$.

potential of an ideal trap is described by

$$
\Phi_{2}=\frac{U_{0} C_{2}}{2 d^{2}}\left(z^{2}-\frac{\rho^{2}}{2}\right)
$$

where $\rho=\sqrt{x^{2}+y^{2}}, U_{0}$ is the voltage between the ring and the end-cap electrodes (see Fig. 1), and $C_{2}$ is a dimensionless constant, which is usually close to unity for hyperbolic Penning traps. The characteristic trap dimension $d$ is related to other characteristic dimensions $\rho_{0}$ and $z_{0}$ (see Fig. 1) via

$$
d=\sqrt{\frac{1}{2}\left(z_{0}^{2}+\frac{1}{2} \rho_{0}^{2}\right)} .
$$

In a real Penning trap where cylindrical symmetry can be still assumed, also higher-order terms exist and can be quantified by the following expansion:

$$
\Phi(r, \theta)=\frac{U_{0}}{2} \sum_{i=2,4, \ldots}^{\infty} C_{i} \frac{r^{i} P_{i}(\cos (\theta))}{d^{i}} .
$$

Here, spherical coordinates $r$ and $\theta$ are used and $P_{i}(\cos (\theta))$ are the Legendre polynomials. The azimuthal angle $\phi$ vanishes due to rotational symmetry. Only even terms are listed, as odd terms are strongly suppressed by mirror symmetry in axial direction. Taking only $i=2$ into account, this expression reduces to the ideal case shown in Eq. (1).

One method to produce such an electric quadrupole potential is a hyperbolically shaped ring electrode and two hyperbolically shaped end-cap electrodes (see Fig. 1) [18]. Another trap geometry being widely used consists of cylindrical electrodes. Additional so-called correction electrodes (also called "guard electrodes" for hyperbolic Penning traps) are implemented. They compensate higher order electric field components $C_{i}$.

In the absence of deviations from the ideal magnetic field and electrostatic potential, the nonrelativistic equation of motion of an ion with charge $q$ and mass $m$ in a Penning trap can be written as

$$
\left(\begin{array}{c}
\ddot{x}^{\prime} \\
\ddot{y}^{\prime} \\
\ddot{z}^{\prime}
\end{array}\right)=\frac{q B_{0}}{m}\left(\begin{array}{c}
\dot{y}^{\prime} \\
-\dot{x}^{\prime} \\
0
\end{array}\right)+\frac{q U_{0} C_{2}}{2 m d^{2}}\left(\begin{array}{c}
x^{\prime} \\
y^{\prime} \\
-2 z^{\prime}
\end{array}\right),
$$

where the primed variables are the coordinates of the ion. Solving this differential equation leads to three independent eigenmotions with frequencies

$$
\begin{gathered}
\nu_{z}=\frac{1}{2 \pi} \sqrt{\frac{q U_{0} C_{2}}{m d^{2}}}, \\
\nu_{ \pm}=\frac{1}{2}\left[v_{\mathrm{c}} \pm \sqrt{v_{\mathrm{c}}^{2}-2 v_{z}^{2}}\right],
\end{gathered}
$$

where $v_{z}$ is the axial, $v_{-}$is the magnetron, and $v_{+}$is the modified cyclotron frequency. The magnetron frequency is independent of the charge-to-mass ratio to lowest order, which allows the comparison of the magnetron frequencies of two independent ions directly with each other with minimal corrections. The free-space cyclotron frequency is defined as

$$
\nu_{\mathrm{c}}=\frac{1}{2 \pi} \frac{q}{m} B_{0} .
$$

In a Penning-trap measurement, only frequencies $v_{ \pm}$and $v_{z}$ are directly measurable but usually the free-space cyclotron frequency is of physical interest. It can be obtained from the eigenfrequencies through, e.g., the invariance theorem [17]:

$$
v_{\mathrm{c}}^{2}=v_{z}^{2}+v_{-}^{2}+v_{+}^{2} .
$$

The hierarchy of the frequencies is $v_{-} \ll v_{z} \ll v_{+}$. Typically, the frequencies range from a few $\mathrm{kHz}$ for $\nu_{-}$up to a few tens of $\mathrm{MHz}$ for $v_{+}$.

\section{IMAGE CHARGE SHIFT}

The trapped ion induces oscillating image charges on the surrounding trap electrodes. While these induced image charges are essential for the detection of the ion's oscillation frequencies [19-22], they cause a systematic shift to the ion's frequencies by generating an undesired electrostatic field $\boldsymbol{E}_{\text {image, }}$ which acts back on the ion and slightly shifts the ion's eigenfrequencies; see Fig. 2. $\boldsymbol{E}_{\text {image }}$ is approximated by

$$
\boldsymbol{E}_{\text {image }}\left(x^{\prime}, y^{\prime}, z^{\prime}\right)=n\left(\mathcal{E}_{x} x^{\prime} \boldsymbol{e}_{x}+\mathcal{E}_{y} y^{\prime} \boldsymbol{e}_{y}+\mathcal{E}_{z} z^{\prime} \boldsymbol{e}_{z}\right),
$$

where the parameters $\mathcal{E}_{i}$ are the so-called electrostatic linear field gradients (LFG) and $n$ is the charge state of the ion. Higher order terms of the induced electric field are also present. They only become relevant for extremely excited ions when the amplitude of their motion becomes comparable to the characteristic trap parameters $z_{0}$ or $\rho_{0}$. The term in parentheses represents the LFG induced by one positive elementary charge. Because of the cylindrical symmetry of the Penning-trap electrodes, the LFG of the ICS should be the same in $x$ and $y$ directions:

$$
\mathcal{E}_{\rho} \equiv \mathcal{E}_{x}=\mathcal{E}_{y} .
$$

The full derivation of the frequency shift due to the ICS can be found in Ref. [23]. The force $\boldsymbol{F}=q \boldsymbol{E}_{\text {image }}$ caused by the image charges on the ion leads to an additional term in the radial equation of motion. The image charges cause a 


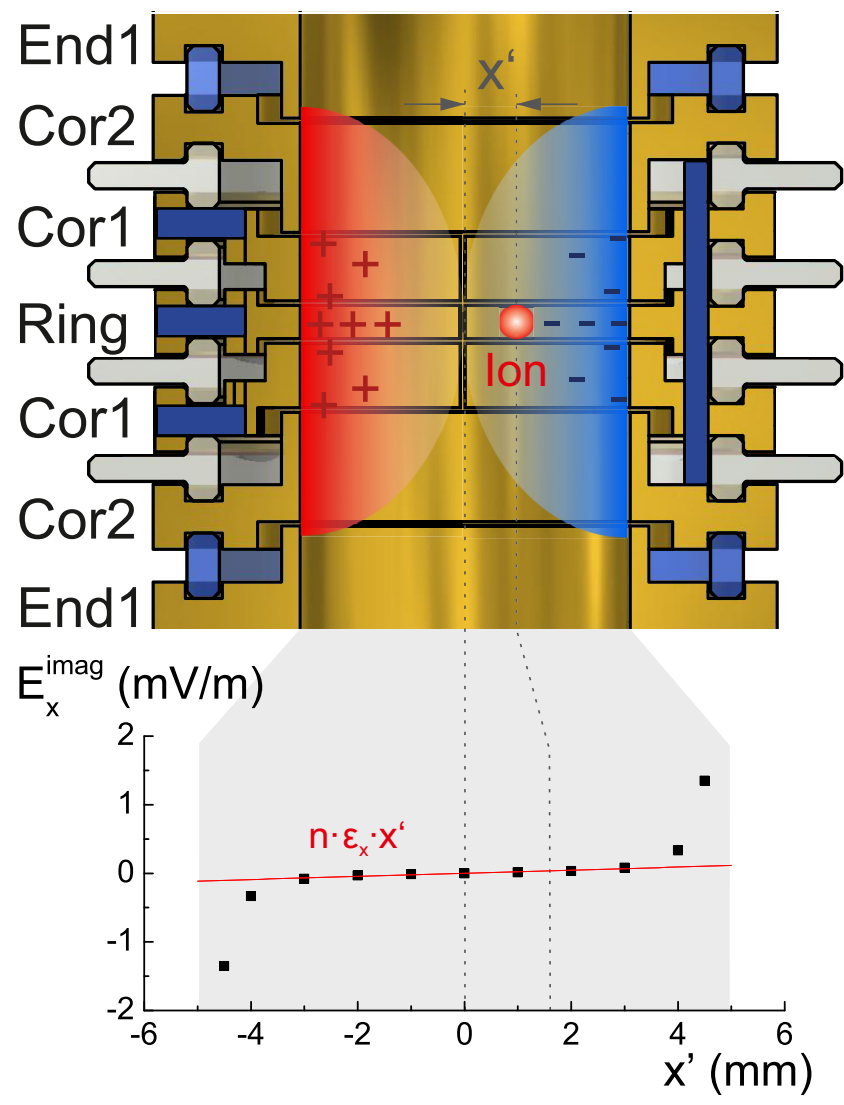

FIG. 2. Illustration of the image charge shift (ICS): In the upper part, the Penning trap of the LIONTRAP experiment is shown with a single positively charged ion shifted in the $x$ direction to position $x^{\prime}$. On the surface of the cylindrical electrodes, the relative change of the induced image charges is shown, which results from the displacement of the ion from the center position. Less induced image charges result in a relative positive change and are sketched in red, and more image charges are sketched in blue. In the lower part, the $x$ component of the electric field simulated by COMSOL MULTIPHYSICS at the ion's position is shown, which is generated by the induced image charges when the ion is placed at position $x^{\prime}$. Uncertainties of the individual points are smaller than the point size. The red line indicates the linear approximation of the induced electric field: $E_{\text {image }, \mathrm{x}}\left(x^{\prime}\right)=n \times \mathcal{E}_{x} \times x^{\prime}$, where $\mathcal{E}_{x}$ is the linear field gradient in $x$ direction.

perturbation $\epsilon_{\rho}$ to the ion motion. This changes the normal radial equation of motion [see Eq. (4)] to

$$
\left(\begin{array}{c}
\ddot{x}^{\prime} \\
\ddot{y}^{\prime}
\end{array}\right)=2 \pi v_{\mathrm{c}}\left(\begin{array}{c}
\dot{y}^{\prime} \\
-\dot{x}^{\prime}
\end{array}\right)+2 \pi^{2} v_{z}^{2}\left(1+\epsilon_{\rho}\right)\left(\begin{array}{c}
x^{\prime} \\
y^{\prime}
\end{array}\right),
$$

where $\epsilon_{\rho}=n \frac{e \mathcal{E}_{\rho}}{4 \pi^{2} m v_{z}^{2}}, q=n \times e$, and $e$ is one elementary charge. It can be assumed that $v_{-} \ll v_{+}$and so the radial frequency shifts becomes

$$
\Delta v_{ \pm}=\mp n \frac{\mathcal{E}_{\rho}}{2 \pi B_{0}} .
$$

It becomes evident from Eq. (12) that the radial sideband frequency $v_{\mathrm{c}}=v_{+}+v_{-}$is immune to the ICS. The axial shift can be calculated as

$$
\Delta v_{z}=-n^{2} \frac{e}{m} \frac{\mathcal{E}_{z}}{8 \pi^{2} v_{z}} .
$$

The shift of the free-space cyclotron frequency, obtained by using the invariance theorem [see Eq. (8)], is then ${ }^{2}$

$$
\Delta v_{\mathrm{c}} \approx n \frac{2 \mathcal{E}_{\rho}+\mathcal{E}_{z}}{4 \pi B_{0}}
$$

The unperturbed free-space cyclotron frequency $v_{\mathrm{c}}$ can be obtained from the measured frequency $\tilde{v}_{\mathrm{c}}$ by

$$
v_{\mathrm{c}}=\tilde{v}_{\mathrm{c}}+\Delta v_{\mathrm{c}}
$$

In cylindrical Penning traps, the electrode surfaces can be approximated as an infinitely long cylinder with radius $\rho_{0}$ for a first-order ICS calculation. In this case, an analytical solution of the ICS exists [24]:

$$
\Delta v_{ \pm}=\mp \frac{q^{2}}{16 \pi^{3} \varepsilon_{0} m \rho_{0}^{3} v_{\mathrm{c}}}
$$

where $\varepsilon_{0}$ is the vacuum permittivity. Consequently, the relative shift of the free-space cyclotron frequency

$$
\Delta v_{\mathrm{c}} / v_{\mathrm{c}} \approx m /\left(4 \pi \varepsilon_{0} B_{0}^{2} \rho_{0}^{3}\right)
$$

shows that the ICS is most relevant in measurements of heavy ions. Furthermore, Eq. (17) illustrates the strong impact of the trap size $\Delta v_{\mathrm{c}} \propto 1 / \rho_{0}^{3}$. For realistic hyperbolic and cylindrical Penning-trap geometries, $\mathcal{E}_{\rho}$ and $\mathcal{E}_{z}$ cannot be determined analytically. In the following, a dedicated experimental approach is described to determine $\Delta v_{\mathrm{c}}$ directly in a Penning trap as well as a dedicated calculation by a finite-element simulation to find $\mathcal{E}_{\rho}$ and $\mathcal{E}_{z}$.

\section{MEASUREMENT OF THE ICS}

Assuming typical Penning-trap parameters, e.g., a magnetic field of 3 to $8 \mathrm{~T}$, a trap radius of 3 to $7 \mathrm{~mm}$, and ion masses of 1 to $250 \mathrm{u}$, the relative ICS in the free cyclotron frequency ranges from a few ppt to several tens of ppb; see Eq. (17). In many cases, this shift is larger than the typical level of statistical precision in state-of-the-art Penning-trap experiments. Aiming for an ICS measurement on a 5\% level or better, for two reasons it is not possible to determine this shift by measuring the three eigenfrequencies of just one single particle: At first, the absolute values of the electric as well as of the magnetic field are not known with sufficient precision to make a precise prediction of the unshifted eigenfrequencies. Second, the measured eigenfrequencies have to be corrected by other systematic shifts, e.g., the tilt of the trap; see Table I. However, all these additional trap parameters can only be determined via eigenfrequency measurements. Therefore, solely

\footnotetext{
${ }^{2}$ Note the change in sign convention here. While the shifts in the previous two equations, (12) and (13), need to be added to the frequencies of the ideal trap-that is, without image charges-in order to yield the actual frequencies in the presence of image charges, the shift in Eq. (14) needs to be added to the measured frequency in order to obtain the free-space cyclotron frequency in the absence of image charges.
} 
TABLE I. Measured magnetron frequency difference of a single proton and a single carbon nucleus, determined in the LIONTRAP setup. The first row indicates the magnetron frequency difference already including the correction of the voltage fluctuations. Various contributions have to be subtracted to derive the sought-after ICS contribution in the magnetron frequency difference: (1) magnetron frequency difference in an ideal trap without any systematics given by Eq. (6), (2) systematic shifts due to the dominant magnetic inhomogeneity $B_{2}$, and (3) systematic shifts due to a tilt and ellipticity of the trap electrodes. The last row indicates the predicted magnetron frequency difference caused by the ICS, as determined by the full COMSOL simulation described in Sec. V (see also Table II) and evaluated for ${ }^{12} \mathrm{C}^{6+}(n=6)$ and $p(n=1)$ respectively, which is in agreement with the measured value.

\begin{tabular}{lccc}
\hline \hline Effect & $\nu_{-}\left({ }^{12} \mathrm{C}^{6+}\right)(\mathrm{mHz})$ & $\nu_{-}(\mathrm{p})(\mathrm{mHz})$ & $\Delta \nu_{-}(\mathrm{mHz})$ \\
\hline$\nu_{-}^{\exp }$ & & & $393.258(98)$ \\
\hline Ideal $\nu_{-}$ & 4770614.107 & 4770223.384 & $390.723(1)$ \\
$B_{2}$ & -0.017 & -0.073 & $0.056(21)$ \\
$\theta_{\text {mag }}, \phi_{\text {mag }}, \epsilon$ & 747.748 & 747.560 & $0.188(37)$ \\
\hline$\nu_{-}^{\operatorname{ex}}($ only ICS $)$ & & & $2.291(126)$ \\
\hline$\nu_{-}^{\text {theo }}($ only ICS $)$ & 2.852 & 0.475 & $2.377(21)$ \\
\hline \hline
\end{tabular}

the linear scaling of the ICS with charge can be measured by varying the number of simultaneously trapped ions of the same species or alternating measurements of ions with different masses in the same trap.

The ICS was first observed in 1989 by Van Dyck et al. [13]. The studied shifts in the magnetron frequency as well as in the modified cyclotron frequency by varying the number of trapped ions of the same species from a very few up to a cloud of about thousand ions. By measuring the linear scaling of the eigenfrequencies with four different ion species, they managed to constrain the ICS with $17 \%$ relative uncertainty. In the year 2000, mass measurements of nonmass doublets started to be strongly limited by the uncertainty of the ICS. For this reason, Van Dyck and colleagues measured the ICS with improved detection techniques [12]. Here, they compared the modified cyclotron frequencies of $24(1){ }^{16} \mathrm{O}^{6+}$ ions to a single ${ }^{16} \mathrm{O}^{6+}$ ion. In an additional measurement, they measured the ICS number scaling with one and two ${ }^{12} \mathrm{C}^{6+}$ ions. Finally, they determined the ICS with a relative uncertainty of $4 \%$, limited by the uncertainty of systematic effects.

To rule out ion-ion interactions, we developed a measurement approach based on two single ions with a large charge difference, which enables ICS measurements with relative uncertainties of a few percent. The relevant trap, referred to as the precision trap (PT) of the LIONTRAP setup [4], is a highly harmonic cylindrical Penning trap with a radius of $5 \mathrm{~mm}$, described in detail in Sec. VI. The PT has two precisely tuned resonators, which pick up the induced image current of a single proton $p$ and a single carbon nucleus ${ }^{12} \mathrm{C}^{6+}$. In this way, both ions can be alternately studied with the same voltage setting and therefore at the same position in the trap and the same magnetic field. The ICS is about three orders of magnitude larger for the radial frequencies (e.g., for the proton: $\left|\Delta v_{ \pm}(p)\right| \approx 0.48 \mathrm{mHz}$ ) compared to the shift in the axial frequency $\left(\left|\Delta v_{z}(p)\right| \approx 0.59 \mu \mathrm{Hz}\right)$, due to the close-to-ideal translational axial symmetry, particularly for cylindrical traps. Based on numerical calculations, which will be introduced in Sec. V, the predicted ICS difference in the radial modes $\left(\left|\Delta v_{ \pm}^{*}\right|=\left|\Delta v_{ \pm}\left({ }^{12} C^{6+}\right)\right|-\left|\Delta v_{ \pm}(p)\right|\right)$ is of the order of $2.3 \mathrm{mHz}$. Since the shift in the axial frequency is so small, the shift in the modified cyclotron mode is nearly equivalent to the shift in the free cyclotron frequency. Limited by the uncertainty of the proton mass as well as by the present magnetic field fluctuations, it is not feasible to measure the ICS with the cyclotron frequency, but only in the magnetron frequency. Aiming for a relative precision of the measured ICS shift of $5 \%$ or less, the uncertainty in the determination of the magnetron frequencies, which are in the order of $4.7 \mathrm{kHz}$, has to be below $85 \mu \mathrm{Hz}$. So far, in our former highprecision experiments, the magnetron frequency has been determined via sideband coupling to an axial detection system in a so-called double-dip spectrum [25,26]. In combination with the required single-dip spectrum, the experiment reached frequency uncertainties of about $50 \mathrm{mHz}$ in the setup after two minutes of averaging the dip- as well as for the doubledip spectrum. While such a precision for $\nu_{-}$is absolutely sufficient for state-of-the-art mass and $g$-factor measurements, the precision needed to resolve the ICS requires a different measurement technique.

\section{A. Ramsey-like phase-sensitive measurement principle}

To reach sub-mHz precision for the magnetron frequency, a phase-sensitive measurement technique was applied. As the magnetron frequency is so small, phase-sensitive techniques, like Pulse and Phase (PnP) or Pulse and Amplify (PnA) $[25,27]$ using the red or blue sideband $v_{\mathrm{rf}}=v_{z} \pm v_{-}$for phase transformation from the magnetron into the axial mode, are problematic. Both of these sideband excitations exhibit a significant component of a direct axial dipolar excitation which parasitically modifies the magnetron phase transformation. Therefore, a Ramsey-like phase-sensitive detection technique has been developed shown in Fig. 3. Here, two short and identical radial dipolar pulses at the frequency of $v_{\mathrm{rf}}=v_{-}$are applied to the singly trapped ion, separated by a precise phase evolution time $T_{\text {evol }}$. The first pulse excites the thermalized magnetron motion of the ion and thus imprints a magnetron phase. Depending on the phase relation between the ion, whose phase evolved for some time $T_{\text {evol }}$, and the fixed phase of the second pulse, the magnetron motion is further enlarged or diminished.

By varying the phase evolution time $T_{\text {evol }}$, the final magnetron radius $r_{-, \text {exc }}$ oscillates periodically with the magnetron frequency:

$$
r_{-, \text {exc }}^{2}\left(T_{\text {evol }}\right)=\frac{r_{-, \text {max.exc }}^{2}}{2}\left\{1+\cos \left[\phi\left(T_{\text {evol }}\right)\right]\right\}+r_{\text {therm }}^{2},
$$

where $\phi\left(T_{\text {evol }}\right)=2 \pi v_{-} T_{\text {evol }}+\phi_{0}$ and in our case $r_{-, \text {max.exc }} \approx$ $274 \mu \mathrm{m}$ (see Fig. 3) for the proton and the carbon ion. The thermal magnetron radius $r_{-}^{\text {therm }}$ amounts to significantly less than $10 \mu \mathrm{m}$ for all cases relevant to this work. In order to detect the excited magnetron radius, the highly harmonic trapping potential is deformed by artificially generated $C_{4}$ and $C_{6}$ terms [see Eq. (3)]

$$
\frac{\Delta v_{z}}{v_{z}}=-\frac{C_{4}}{C_{2}} \frac{3}{2 d^{2}} r_{-, \mathrm{exc}}^{2}+\frac{C_{6}}{C_{2}} \frac{45}{16 d^{4}} r_{-, \mathrm{exc}}^{4},
$$




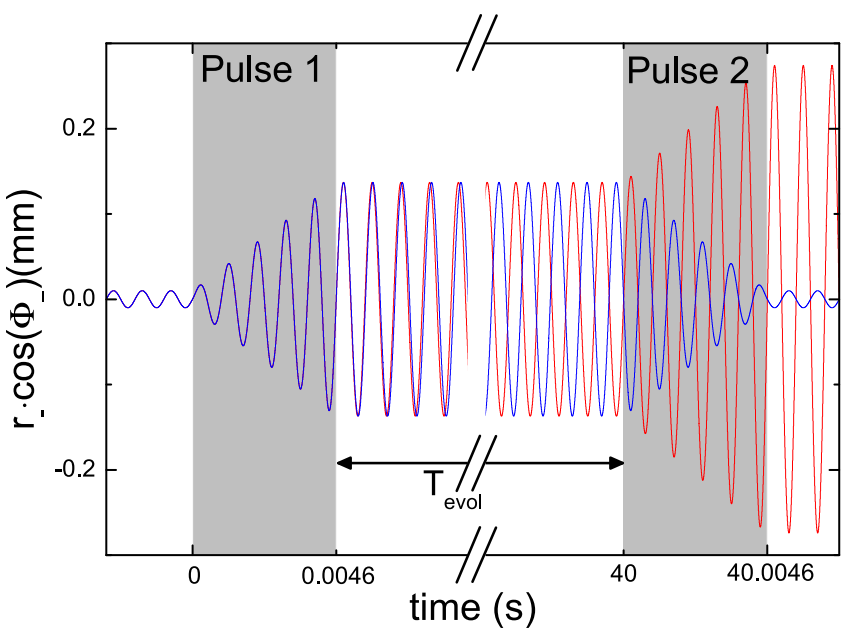

FIG. 3. Ramsey-like excitation scheme for a high-precision measurement of the magnetron frequency. The pattern contains two identical radial dipolar pulses (gray shaded areas) with pulse lengths of $4.6 \mathrm{~ms}$ each and excitation frequencies of $v_{\mathrm{rf}}=v_{-}$, separated by a phase evolution time $T_{\text {evol }}$. The first pulse excites the thermalized magnetron motion of the ion $\left(r_{-}^{\text {therm }}<10 \mu \mathrm{m}\right)$ to $r_{-}^{\mathrm{exc}} \approx 137 \mu \mathrm{m}$ and thus imprints a magnetron phase. After the second pulse, the two extreme cases are shown: maximum excitation in red and basically no excitation in blue.

where the measured potential coefficients are $C_{2}=$ $-0.5997, \quad C_{4}=-0.00223(18), \quad C_{6}=0.014(4), \quad$ and $\Delta v_{z}=v_{z}(\mathrm{exc})-v_{z}($ cold $)$ is the axial frequency difference at the deformed trapping voltage. Here, $v_{z}(\mathrm{exc})$ is measured after the application of the Ramsey-like excitation scheme and $v_{z}$ (cold) is measured without any excitation. At maximum magnetron excitation, the axial frequency shift for the proton is $\Delta v_{z}^{\max }(p)=-12.3(9) \mathrm{Hz}$ at an absolute frequency of $v_{z}(p)=739865 \mathrm{~Hz}$. In comparison with other energy-dependent axial frequency shifts, the $C_{4}$ term dominates the axial frequency shift by $97 \%$. For reliable switching between the harmonic and the deformed voltage setting, the required voltage modification is performed by voltage channel mixing via a relay and a voltage divider, so that the setting of the high-precision voltage source is not touched.

The magnetron frequency is found by measuring the magnetron phase at two different phase evolution times, e.g., $T_{\mathrm{evol}, 1}=0.1 \mathrm{~s}$ and $T_{\mathrm{evol}, 2}=40 \mathrm{~s}$ :

$$
\nu_{-}=\frac{1}{2 \pi} \frac{\phi\left(T_{\mathrm{evol}, 1}\right)-\phi\left(T_{\mathrm{evol}, 2}\right)}{T_{\mathrm{evol}, 1}-T_{\mathrm{evol}, 2}} .
$$

One single magnetron phase is determined by the measurement of one period of these Ramsey-like oscillations; see Fig. 4.

Equations (18) and (19) are used to fit $\phi_{0}$ in a one parameter fit, where the maximal magnetron radius is determined via

$$
\begin{aligned}
r_{-, \text {max.exc }}^{2}= & \frac{4}{15} \frac{C_{4}}{C_{6}} d^{2} \\
& +\sqrt{\left(\frac{4}{15} \frac{C_{4}}{C_{6}} d^{2}\right)^{2}+\frac{16}{45} \frac{C_{2}}{C_{6}} d^{4} \frac{\Delta \nu_{z}^{\max }}{v_{z}}} .
\end{aligned}
$$

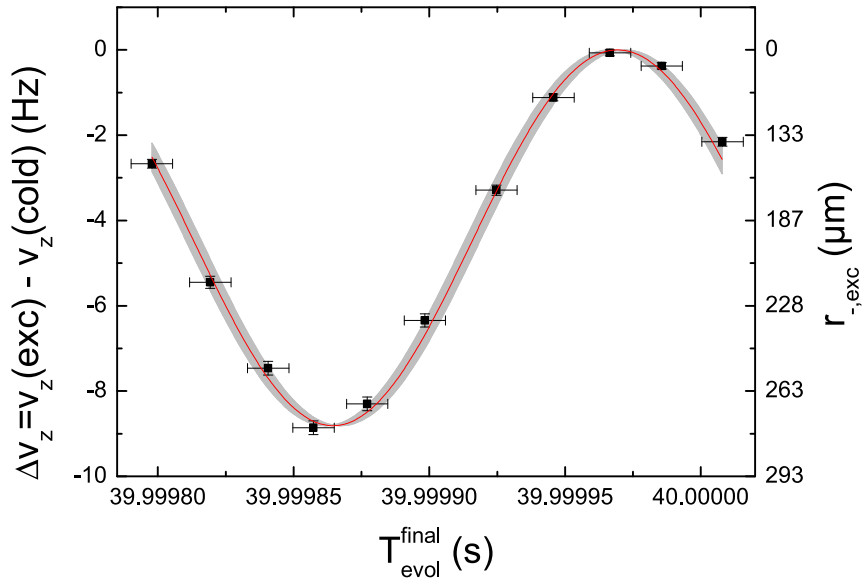

FIG. 4. One example of the determination of the magnetron phase by axial frequency shifts. Here, the Ramsey-like excitation scheme has been applied on a single ${ }^{12} \mathrm{C}^{6+}$ ion at 11 different phase evolution times $\left(T_{\mathrm{evol}}=39.9998, \ldots, 40 \mathrm{~s}\right)$. On the $y$ axis, the axial frequency differences $\Delta v_{z}=v_{z}($ exc $)-v_{z}$ (cold) are plotted. The error bars are given by the uncertainties of the axial frequencies measured at the deformed voltage setting $\left[\delta\left(\Delta v_{z}\right)=70 \mathrm{mHz}\right]$ and the intrinsic thermal distribution of the magnetron radius. On the $x$ axis, the final phase evolution times are indicated, which include voltage corrections; see Appendix A. The corresponding error bars of the voltage corrected phase evolution time $\delta T_{\text {evol }}^{\text {final }}=2 T_{\text {evol }} \delta v_{z}^{\text {harm.volt }} / \nu_{z}^{\text {off }}$ are given by the axial frequency uncertainties measured at the harmonic voltage setting $\left(\delta v_{z}^{\text {harm.volt }}=50 \mathrm{mHz}\right)$. In this plot, the magnetron phase at $39.9998 \mathrm{~s}$ and modulo $360^{\circ}$ is determined by the fit indicated as a red line with the gray confidence interval: $\phi\left(T_{\text {evol }}^{\text {final }}=39.9998 \mathrm{~s}\right)=249(5)^{\circ}$.

Here, the miniscule effect of the thermal radius has been neglected. Moreover, in $\phi\left(T_{\text {evol }}\right)=2 \pi \nu_{-}^{\text {init }} T_{\text {evol }}+\phi_{0}$ the magnetron frequency $\left(v_{-}^{\text {init }}\right)$ is measured at the beginning of the measurements campaign and set to $v_{-}^{\text {init }}(p)=4770.96 \mathrm{~Hz}$ and $\nu_{-}^{\text {init }}\left({ }^{12} \mathrm{C}^{6+}\right)=4771.353 \mathrm{~Hz}$.

\section{B. Measurement cycle}

In Fig. 5, the complete measurement cycle is illustrated. In the beginning of each cycle, either the proton or the carbon nucleus is randomly chosen and transported into the PT, while the other ion is confined in an adjacent storage trap (ST). During the ion transport, different voltages are applied, which lead to temperature fluctuations in the voltage divider of the voltage source. A certain relaxation time is required to reduce the involved temperature changes in the voltage divider. This is followed by some precooling of the ions' magnetron and modified cyclotron motion. Then $2 \times 11$ Ramsey cycles of different phase evolution times are applied in random order. The phase evolution times are predefined and range from 0.1 to $40 \mathrm{~s}$ (some at $20 \mathrm{~s}$ ); see Fig. 4.

In the beginning and at the end of these Ramsey cycles, three axial dips are recorded: (1) with thermalized magnetron mode and harmonic potential, for the correction of voltage fluctuations as described in Appendix A, (2) with thermalized magnetron mode and deformed potential $\left[v_{z}(\right.$ cold $\left.)\right]$, and (3) with maximally excited magnetron mode and deformed 


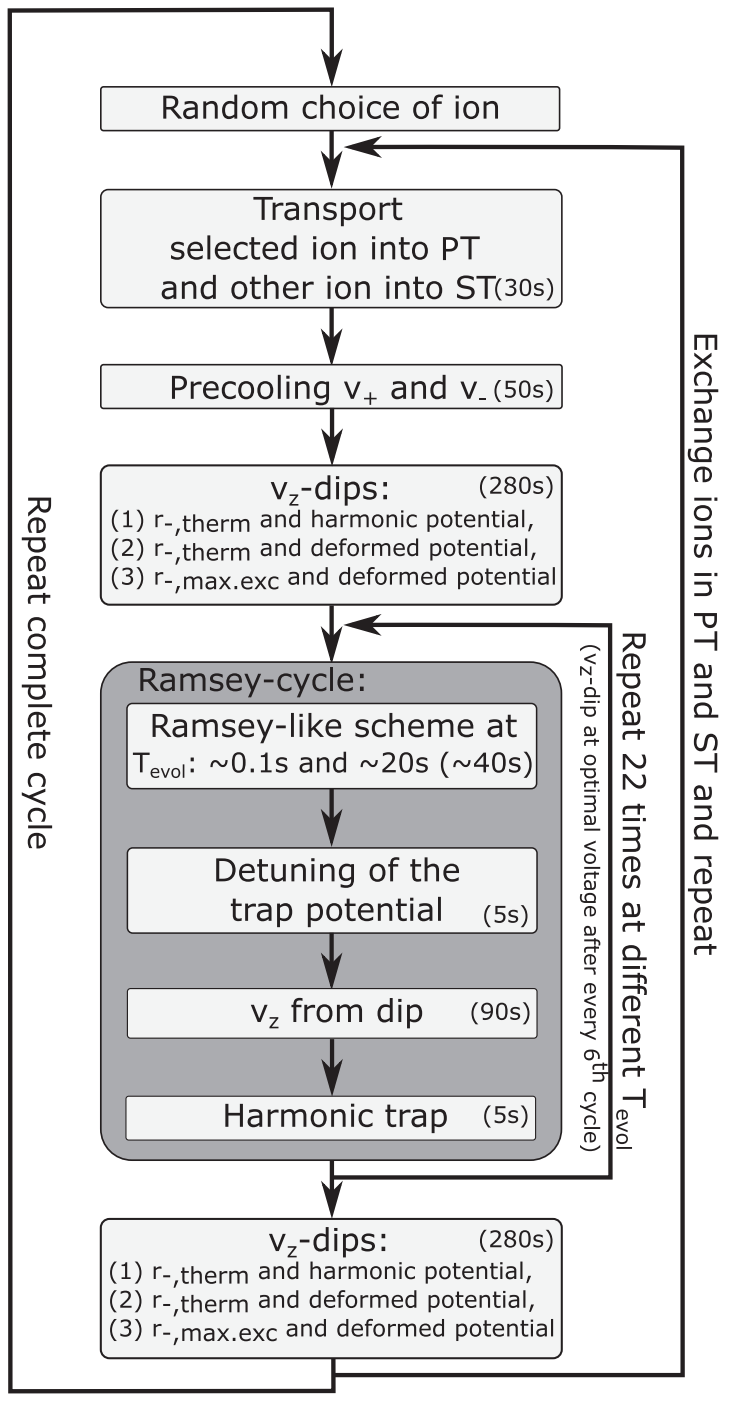

FIG. 5. Illustration of the measurement cycle. One complete measurement cycle takes $140 \mathrm{~min}$ and reaches a statistical precision of about $480 \mu \mathrm{Hz}$ for the magnetron frequency difference. In total, 120 cycles have been recorded over a period of 23 days, where 73 cycles feature a $T_{\mathrm{evol}, 2}$ of $40 \mathrm{~s}$ and the others $20 \mathrm{~s}$.

potential for the determination of the maximal axial shift $\left(\Delta v_{z}^{\max }\right)$. After every sixth Ramsey cycle, the axial dip is measured again to correct the magnetron frequency for voltage drifts and fluctuations in the final analysis $\left(v_{z}^{\text {interpl }}\right)$, when the ion magnetron mode has thermalized and the potential is harmonic. Finally, the complete procedure is repeated with the other ion.

\section{Results of the ICS measurement}

Averaging over 120 measurement cycles (see Fig. 6) yields our experimental result:

$$
\begin{aligned}
\Delta v_{-}^{\exp } & =v_{-}\left({ }^{12} \mathrm{C}^{6+}\right)-v_{-}(p) \\
& =393.258(61)(77) \mathrm{mHz}
\end{aligned}
$$

where the two numbers in brackets indicate the statistical and systematic uncertainties, respectively. The systematic uncertainty arises from the correction of voltage fluctuation.

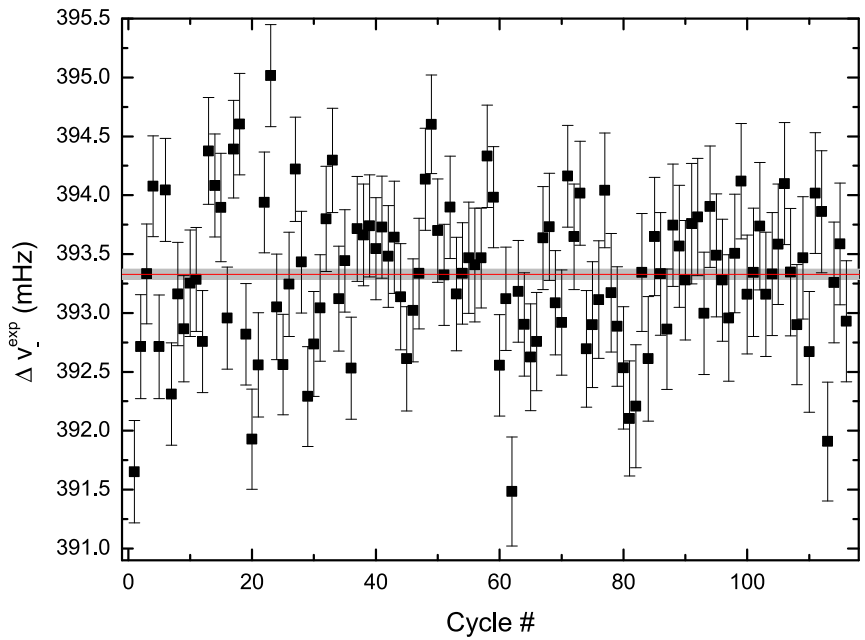

FIG. 6. Magnetron frequency differences of all 120 measurement cycles. The red line indicates the average value and the gray band its $43 \mu \mathrm{Hz}$ statistical uncertainty. The fit of the data returns a $\chi^{2}$ of 2 . This might be explained by tiny axial frequency fluctuations caused by tilt fluctuations. Since a disentanglement between voltage and tilt fluctuations based on the axial frequency is impossible, the tilt fluctuations are interpreted as voltage fluctuations, which leads to false corrections of the corresponding magnetron frequencies. To account for this, the statistical uncertainty is enlarged by a factor of $\sqrt{2}$ suggested by the reduced $\chi^{2}$. The total statistical uncertainty amounts to $61 \mu \mathrm{Hz}$. For details, see text and Appendix A.

There, the axial frequency ratio $R=v_{-}\left({ }^{12} \mathrm{C}^{6+}\right) / v_{-}(p)$ has to be calculated with high precision, which is explained in Appendix A.

In order to isolate the ICS from the measured magnetron frequency difference in Eq. (22), the following contributions have to be subtracted. First, the magnetron frequencies of the two ions in an ideal trap without any systematic shifts are determined by Eq. (6): $v_{-}($ideal $)=0.5\left(v_{\mathrm{c}}-\sqrt{v_{\mathrm{c}}^{2}-2 v_{z}^{2}}\right)$. Here, the modified cyclotron and axial frequency of the proton are measured, whereas the corresponding frequencies of the carbon ion are derived from the measured proton frequencies and the given masses. The resulting ideal magnetron frequency difference has to be further corrected for several systematic shifts generated by the residual inhomogeneity of the magnetic field, the tilt between the magnetic and electric field axis characterized by $\theta_{\mathrm{mag}}$ and $\phi_{\mathrm{mag}},{ }^{3}$ and the elliptic deformation $(\epsilon)$ of the electric field. While the residual cylindrically symmetric electrostatic field imperfections with the optimized potential coefficients $C_{4}(\mathrm{opt})=0(1) \times 10^{-6}$ and $C_{6}(\mathrm{opt})=6.45(16) \times 10^{-4}$ are negligible at the current level of precision, the leading-order magnetic inhomogeneity $B_{2}=-0.270(15) \mathrm{T} / \mathrm{m}^{2}$ together with $T_{z}(p)=3.4(1.0) \mathrm{K}$ and $T_{z}\left({ }^{12} \mathrm{C}^{6+}\right)=6.4(1.0) \mathrm{K}$ contribute a sizable frequency shift (see Table I). The tilt and ellipticity contributions are not easily disentangled unambiguously, ${ }^{4}$ but preliminary

\footnotetext{
${ }^{3}$ The angles $\theta_{\text {mag }}$ and $\phi_{\text {mag }}$ are defined according to the definition in Eq. (2.72) of Ref. [17].

${ }^{4}$ The angle and its uncertainty has been determined by the following relation: $v_{\mathrm{c}}^{*}-v_{\mathrm{c}}=v_{-}\left(9 / 4 \theta_{\mathrm{mag}}^{2}-1 / 2 \epsilon^{2}\right)[28]$, with $v_{\mathrm{c}}^{*}=v_{+}+v_{-}$,
} 
TABLE II. First, the characteristic trap dimensions $\rho_{0}$ and $z_{0}$ are presented. All values are given at a temperature of $4 \mathrm{~K}$. A manufacturing tolerance of $\pm 10 \mu \mathrm{m}$ is assumed for all trap dimensions. Additionally, the magnetic field strengths and the types of Penning traps are displayed. This is followed by a comparison of numerical calculations, semianalytical calculations, and measurements of the ICS for three different high-precision Penning-trap experiments and three different approaches: (1) Semianalytical calculation with simplified geometry, denoted as (Porto); (2) numerical calculation with simplified geometry, denoted as (COMSOL-Porto), and (3) numerical calculation with full geometry, denoted as (COMSOL-full). Uncertainties for the LFGs and the free-space cyclotron frequency shift in the scenario (COMSOL-full) are separately listed: ()$_{\text {sim }}$ and () $)_{\text {geo }}$.

\begin{tabular}{|c|c|c|c|}
\hline Properties & FSU-Trap & THe-Trap & LIONTRAP \\
\hline Type & Hyperb. & Hyperb. & Cylind. \\
\hline$\rho_{0}\left(z_{0}\right)(\mathrm{mm})$ & $6.96(6.00)$ & $2.77(2.29)$ & 5 \\
\hline$d(\mathrm{~mm})$ & 5.501 & 2.124 & 5.107 \\
\hline$B_{0}(\mathrm{~T})$ & 8.529 & 5.915 & 3.764 \\
\hline $\mathcal{E}_{\rho}($ Porto $)$ in $\mu \mathrm{V} / \mathrm{m}^{2}$ & $2818.69(15)$ & $42458(13)$ & $11551(2)$ \\
\hline $\mathcal{E}_{\rho}(\mathrm{COMSOL}-$ Porto $)$ in $\mu \mathrm{V} / \mathrm{m}^{2}$ & $2816.8(1.6)$ & $42405(37)$ & $11545(49)$ \\
\hline $\mathcal{E}_{\rho}(\mathrm{COMSOL}$-full $)$ in $\mu \mathrm{V} / \mathrm{m}^{2}$ & $2839.7(1.6)(11.5)$ & $42604(37)(366)$ & $11230.0(48.5)(84.0)$ \\
\hline $\mathcal{E}_{z}$ (Porto) in $\mu \mathrm{V} / \mathrm{m}^{2}$ & $4200.14(2.0)$ & $80793(25)$ & $0.00(1.53)$ \\
\hline $\mathcal{E}_{z}\left(\right.$ COMSOL-Porto) in $\mu \mathrm{V} / \mathrm{m}^{2}$ & $4198.8(2.1)$ & $80719(69)$ & $0(2)$ \\
\hline $\mathcal{E}_{z}(\mathrm{COMSOL}-$ full $)$ in $\mu \mathrm{V} / \mathrm{m}^{2}$ & $4225.7(2.5)(21.3)$ & $79726(69)(1322)$ & $8.1(3.1)(2.2)$ \\
\hline$\Delta v_{\mathrm{c}}($ Porto $)$ in $\mu \mathrm{Hz} / n$ & $91.7850(34)$ & $2229.37(49)$ & $488.416(58)$ \\
\hline$\Delta v_{\mathrm{c}}(\mathrm{COMSOL}-\mathrm{Porto})$ in $\mu \mathrm{Hz} / n$ & $91.738(36)$ & $2225.1(1.4)$ & $488.7(2.1)$ \\
\hline$\Delta v_{\mathrm{c}}(\mathrm{COMSOL-full})$ in $\mu \mathrm{Hz} / n$ & $92.416(37)(292)$ & $2218.8(1.4)(20.3)$ & $475.4(2.1)(3.6)$ \\
\hline$\Delta v_{\mathrm{c}}$ (measurements) in $\mu \mathrm{Hz} / n$ & & $2230(90)[12]$ & $471.9(23.9)$ \\
\hline
\end{tabular}

measurements indicate $\epsilon=0.011_{-11}^{+4}$ and $\theta_{\text {mag }}=0.56_{-8}^{+7 \circ}$, whereas the insignificant angle $\phi_{\text {mag }}$ cannot be constrained. After subtracting the above-mentioned contributions from the measured frequency difference, we obtain the sought-after ICS:

$$
\Delta \nu_{-}^{\exp }(\text { only ICS })=2.291(61)(111) \mathrm{mHz}
$$

This result is in agreement with the calculations presented in this work; see Table II. The uncertainty of the tilt and the ellipticity shift contribute in the uncertainty of the voltage fluctuation, $\delta\left(\Delta v_{-}\right)_{\theta, \phi, \epsilon}=73 \mu \mathrm{Hz}$ (see Table IV, Appendix A), and in the uncertainty of the magnetron frequency difference, $\delta\left(\Delta v_{-}\right)_{\theta, \phi, \epsilon}=37 \mu \mathrm{Hz}$ (see Table I). These uncertainties are highly correlated so that in the final systematic uncertainty this effect contributes with $\delta\left(\Delta \nu_{-}\right)_{\theta, \phi, \epsilon}=110 \mu \mathrm{Hz}$. The uncertainty of the quadratic magnetic inhomogeneity leads to an uncertainty of the voltage fluctuation (see Table IV, Appendix A) and to an uncertainty of the magnetron frequency difference (see Table I). Both uncertainties are directly correlated and cancel each other out. Thus, the ICS calculations can be confirmed on a modest $5 \%$ level.

\section{SIMULATIONS OF THE ICS}

As detailed above, a sufficiently precise measurement of the ICS requires a specially tailored experimental setup, e.g., two axial resonators matching the axial frequencies of two different ions for the same voltage setting and a dedicated construction to cancel the trap tilt. As most Penning-trap experiments do not exhibit these special features, a possibility to directly simulate the size of the ICS and thus correct for

$v_{\mathrm{c}}=\sqrt{v_{+}^{2}+v_{\mathrm{z}}^{2}+v_{-}^{2}}$, and the assumption of an upper bound of $|\epsilon| \leqslant$ 0.015 . Angles are entered in radians in all formulas in this work. the shift is of great importance. For the very first ICS measurement in 1989 [13], an extremely simple analytical model provided a successful confirmation of the measured ICS. In Ref. [13], the hyperbolic geometry of the electrodes has been approximated by a sphere. Subsequently in 2000, Häffner derived an analytical first-order approximation for cylindrical Penning traps [24] by approximating the segmented electrodes as an infinitely long cylinder, as in Eq. (17). Soon afterward in 2001, an elaborate semianalytical model was developed by Porto, which was directly applied on hyperbolic traps [14]. In Appendix $\mathrm{C}$, this method has been reanalyzed in detail. Here, two minor flaws of this semianalytical model have been recognized and corrected. In 2013, a further refined analysis has been modeled by Kretzschmar and others considering also vertical and horizontal slits of cylindrical traps [15]. All these methods above are limited by the fact that complicated shapes of the electrodes have to be simplified in order to calculate the ICS theoretically. Finite-element simulations as discussed below overcome these problems with the trade-off of high demand in computational power.

\section{A. Finite-element approach}

Only numerical calculations of the ICS are able to consider the full electrode geometries of all kinds of Penning traps. The goal of such simulations is the determination of the LFG $\mathcal{E}_{i}$ introduced in Eq. (9). As a simulation tool, the program COMSOL MULTIPHYSICS is chosen, which is a commercial general-purpose platform software for modeling various physical applications. The functionality of the basic software covers the finite-element method of solving differential equations in complex geometries [29]. The software can be expanded by the AC/DC module, which covers solving of electrostatic problems. 


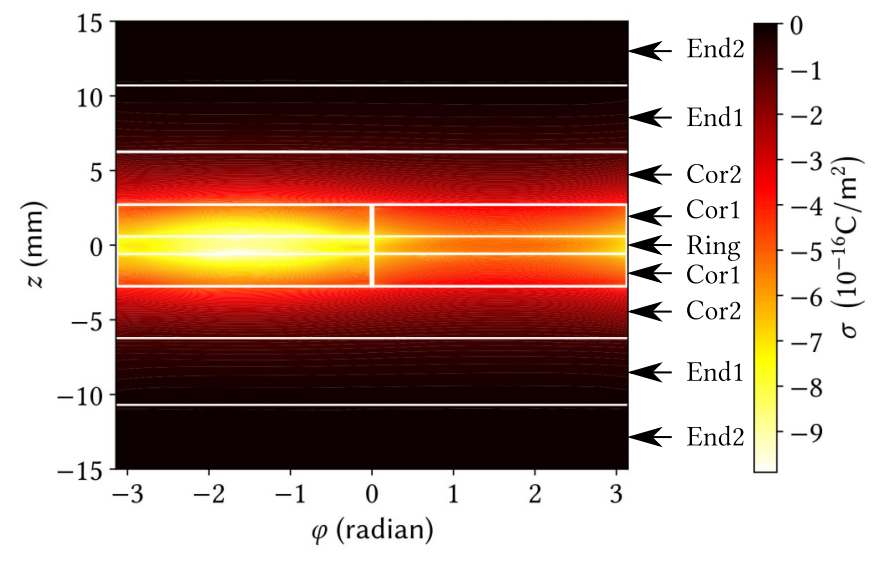

FIG. 7. Illustration of the numerical calculation of the surface charge density $\sigma$ [see Eq. (24)] on the electrodes of a cylindrical Penning trap induced by a single charged particle at $x^{\prime}=-0.5 \mathrm{~mm}$. A projection of the surfaces of the cylindrical measurement trap of the LIONTRAP experiment into the plane is shown.

After modeling the trap geometry within the software, physical parameters can be set, e.g., the position and charge of the pointlike ion and the Dirichlet boundary conditions at the perfectly conducting electrode surfaces. For the numerical solution of the static Maxwell equations, the initially implemented shape of the electrodes and the space between them is approximated by small elements called "mesh." Minimum and maximum sizes of the mesh elements can be set for the algorithm which creates the mesh. The algorithm chooses automatically the minimum size for areas with detailed structures, as for example the edges of the electrodes. On the meshed electrode surfaces, the numerical solution provides the sought-after induced surface charge density $\sigma$; see Fig. 7. In a next step, the electric field generated by the induced image charges on the electrode surfaces is calculated at the position $\boldsymbol{x}^{\prime}$ of the ion:

$$
\boldsymbol{E}_{\text {image }}\left(\boldsymbol{x}^{\prime}\right)=-\int \frac{\sigma\left(x, y, z, x^{\prime}, y^{\prime}, z^{\prime}\right)}{4 \pi \varepsilon_{0}} \frac{\boldsymbol{x}-\boldsymbol{x}^{\prime}}{\left|\boldsymbol{x}-\boldsymbol{x}^{\prime}\right|^{3}} d A .
$$

Here, the unprimed letters $(x, y, z)$ are the integration variables at the electrode surfaces $A(d A=d x d y d z)$. In the simulation, the ion is moved along $x^{\prime}$ from $-0.5 \mathrm{~mm}$ to $+0.5 \mathrm{~mm}$ in $0.05-$ mm steps, where $y^{\prime}=z^{\prime}=0$. For each step, the surface charge density is simulated and afterward the electric field strength is calculated at the position of the ion by Eq. (24). The resulting electric field strengths at the 21 different ion positions are fitted by an odd polynomial function. The linear coefficient of these fit polynomials is the searched after $\mathcal{E}$; see Eq. (9).

Four systematic uncertainties must be taken into account for the final result:

(1) Numerical uncertainty. The mesh is an approximation of the real geometry. The size of the smallest mesh element specifies the accuracy of the trap geometry in the simulation. The smaller the size, the better the geometry is described. The simulation mostly converges to a value, which is slight off of the unknown correct result. In order to test the simulation for a systematic deviation stemming from the chosen mesh size, a geometry with an analytic solution, which is close to the investigated case, should be simulated. Further details can be found in Appendix B.

(2) Fit uncertainty. The electric field created by the image charges at the different ion positions has to be described by a polynomial to retrieve $\mathcal{E}$. The used fit functions give an uncertainty of the result, as well. This cannot be disentangled from the uncertainty introduced above, even though their origins are different. Both 1 and 2 are therefore summed up in the uncertainty denoted with ( $)_{\text {num }}$.

(3) Geometric uncertainty. The geometric uncertainty is caused by tolerances in the manufacturing process. The real electrodes can deviate from the design value by up to $\pm 10 \mu \mathrm{m}$. To approximate the influence of the geometry on the result, the worst-case scenario is considered. The characteristic trap parameters $\rho_{0}$ and $z_{0}$ are enlarged by $10 \mu \mathrm{m}$. The guard electrodes are shifted up- and outward by $10 \mu \mathrm{m}$, too. The uncertainty stemming from this is denoted by ()geo.

(4) Higher order terms. The effect of higher order terms in the image charge field can become sizable in the case of large motional radii or if the electrostatic center of the trap is shifted with respect to the geometrical center. Reasons for this shift can be, for example, alignment imperfections or patch potentials. The higher order terms of the image charge field can be parameterized by

$$
\vec{E}_{\text {image, h.o. }}=n \times \mathcal{E}_{\text {h.o. }} \times \rho^{3} \times \vec{e}_{\rho} .
$$

The prefactor $\mathcal{E}_{\text {h.o. }}$ is the scaling of the higher order (h.o.) effect. Effects in the $z$ direction are neglected as they are typically strongly suppressed by the usage of the invariance theorem and in the case of cylindrical traps additionally by the inherent symmetry.

If, without loss of generality, a parasitic shift $\hat{\rho}_{0}$ of the trap center in the $\rho$ direction is assumed, this leads to the leading order of an effective, additional image charge field of

$$
\vec{E}_{\text {image, h.o. eff. }}=2 n \times \mathcal{E}_{\text {h.o. }} \times \hat{\rho}_{0}^{2} \times \rho \times \vec{e}_{\rho} .
$$

Independent measurements show that it is reasonable to assume $\hat{\rho}_{0}<50 \mu \mathrm{m}$ [30]. Therefore, the higher order terms contribute to the ICS by less than relative $10^{-2}$ and are thus negligible compared to manufacturing tolerances.

\section{B. Semianalytical approach}

The finite-element method has become an efficient tool due to the increase in computer processing power in recent years. Even geometries with many details can be simulated precisely in three dimensions, in a reasonable amount of time and with good precision. In the past, semianalytical approaches were chosen, which require less computing power but considerably more effort in setting the right boundary conditions and in setting simplifications of the simulated geometry. Porto developed such a concept for the ICS in 2001 [14]. In the following, a short introduction to this approach is given. A more detailed explanation can be found in Appendix C.

In order to calculate the electric field of the image charges numerically efficiently, an approach using a Green's function is chosen [see Eq. (C2)]. The solution of this equation is a sum of associated Legendre polynomials which are scaled with prefactors [see Eq. (C4)]. The final value of the prefactors 


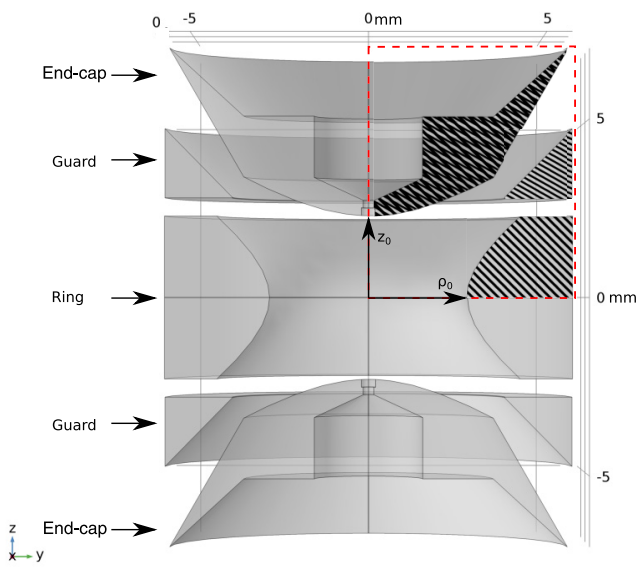

(a) A semitransparent cut-view of the electrode geometry of THe-Trap.

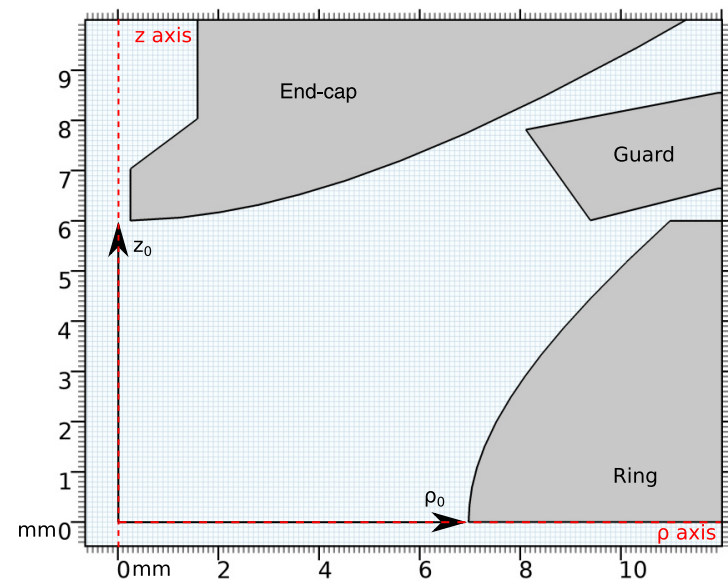

(c) FSU-Trap

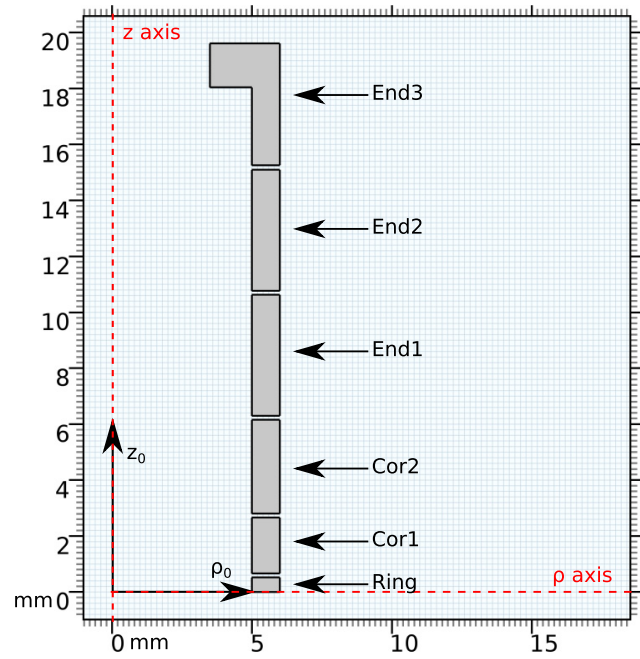

(b) LIONTRAP

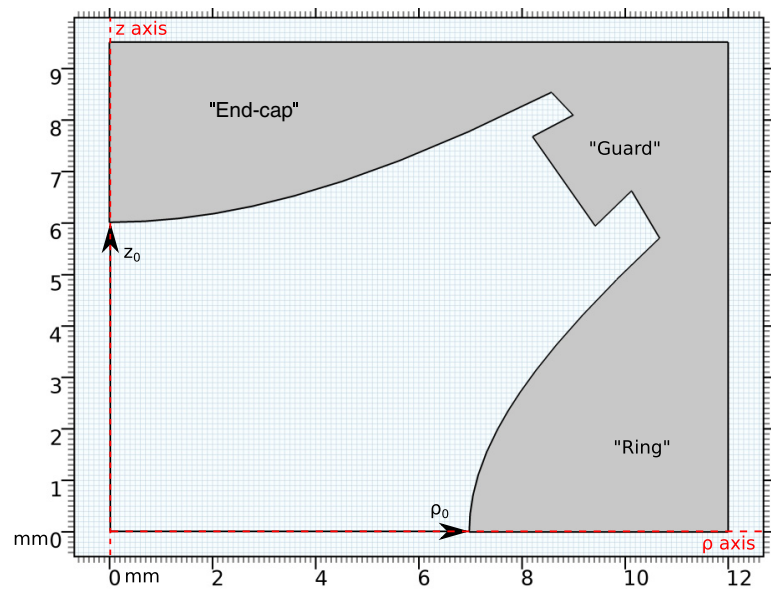

(d) Porto-Trap

FIG. 8. Showing the four different simulated trap geometries. The values of the characteristic trap dimensions $z_{0}$ and $\rho_{0}$ can be found in Table II. (a) The mass-measurement trap of the THe-Trap experiment consists of a hyperbolically shaped ring electrode, two end-cap electrodes, and two guard electrodes. The splitting of the guard electrodes is not taken into account for the ICS, because this effect is negligible. In general, due to the rotational symmetry along the $z$ axis and the mirror symmetry at $z=0$, the full information of the trap geometry can be obtained from looking at one quarter of a cut [see the hatched area surrounded by the red dashed line in panel (a)] of the whole trap. For the other three traps, therefore, only this quadrant is shown. (b) The precision trap (PT) of the LIONTRAP [31] experiment is a cylindrical Penning trap and consists of 11 electrodes. The inner three electrodes (the Ring and Cor1) are all azimuthally split into two pieces, which allows applying different radio-frequency multipoles. The gaps between the electrodes and the split segments is $140 \mu \mathrm{m}$. (c) The FSU-Trap is a hyperbolic Penning-trap mass spectrometer. Similar to THe-Trap, it consists of a ring, two end caps, and two guard electrodes. Like in panel (a) (THe-Trap), the splitting of the guard electrodes into two segments is also not considered. (d) The Porto-Trap is a simplified version of the FSU-Trap geometry with the same characteristic dimensions $z_{0}$ and $\rho_{0}$. In this approximation, holes in the end-cap electrodes and slits are ignored. Furthermore, the guard electrodes are simplified and the space between the electrodes is closed. These modifications are necessary to make the semianalytical treatment possible.

is determined by a minimization approach [see integral in Eq. (C8)]. This integral can mostly be only calculated numerically and thus this approach is called semianalytical. In order to be able to carry out this integral at all, the boundary conditions must be carefully selected. This case means that the integral only runs in a small range of $z$ and $\rho$. Setting the ground potential far away would lead to a diverging integral. Therefore, the holes in the end caps and the space between the guard and the other electrodes is closed. This leads to an increase of the surface of the electrodes facing the ion. In addition, the shape of the guards is simplified to symmetrize the bounds of the integrals which need to be carried out. These changes in the geometry can be seen in the comparison of actual trap geometry in Fig. 8(c) and the geometry for the semianalytical treatment in Fig. 8(d).

\section{STUDIED PENNING TRAPS}

In the following, the ICS is studied for three specific experimental setups: THe-Trap, LIONTRAP, and FSU-Trap; 
see Fig. 8. Furthermore, a simplified Penning trap geometry, the so-called Porto-Trap, is studied, which has been introduced by Porto for a semianalytical calculation of the ICS in the FSU-Trap [14]. The Porto-Trap is analyzed by a revised semianalytical approach (see Appendix C) and by a finiteelement simulation. This offers the chance to compare the new semianalytical result with the result achieved by Porto and to perform a systematic test of the finite-element simulation.

\section{COMPARING DIFFERENT ICS DETERMINATION METHODS}

In this chapter, ICS results of the finite-element simulation are compared with the most precise ICS measurements and the semianalytical treatment; see Table II. For all three geometries, the LFGs $\mathcal{E}_{\rho}, \mathcal{E}_{z}$, and the final shift in the cyclotron frequency have been calculated in three ways: (1) Semianalytical calculations based on the approach by Porto and therefore denoted as "Porto." (2) In order to compare the numerical simulations with the semianalytical predictions, also the simplified geometries have been calculated in COMSOL MULTIPHYSICS denoted as "COMSOL-Porto". This serves as a crosscheck. (3) The complete geometries without simplifications have been calculated in COMSOL MULTIPHYSICS for the final determination of the ICS, entitled as "COMSOL-full."

The relative deviation of less than $0.2 \%$ between the semianalytical and finite-element approaches shows how well the numerical approach works. The difference of $2.7(4) \%$ between the simplified "COMSOL-Porto" and the model containing all details, which are relevant for the image charge shift "COMSOL-full" in the LIONTRAP case illustrates the importance of taking geometric subtleties into account. Here, $2.2(4) \%$ are caused by the horizontal slits and $0.5(4) \%$ by the azimuthal slits in the Cor1 electrodes and the ring electrode.

Further improvements are not limited by the available computational power. The largest uncertainty in the finiteelement approach comes from the manufacturing tolerances. In total, the ICS calculation by the FEM simulation has an uncertainty of $1 \%$, which is a factor of four better than the best measurement so far. The effect of deviation from cylindrical symmetry, resulting in first order in an effective ellipticity of the image charge field, has been estimated and is negligible on the current level of precision.

The new ICS measurement reaches precision similar to the measurement by Van Dyck. The ICS measurement of the LIONTRAP experiment confirms the prediction by the simulation. As it agrees with both the previous method by Van Dyck and the new (see Sec. IV), the simulation serves as an excellent cross-check and shows its predictive power.

\section{CONCLUSION AND OUTLOOK}

The ICS is one of the leading sources of systematic uncertainty in modern high-precision Penning-trap experiments with uncertainties below $10^{-10}$. In this paper, we demonstrate that modern finite-element simulations confirm the result of the semianalytical treatment of the ICS, as introduced by Porto. Both methods reach the same precision, if they are applied to the same, simplified geometry. Moreover, finite-element simulations show that slits and holes in the electrodes shift the result by up to $2.7 \%$.

Numerical uncertainties of finite-element simulations have been significantly reduced. For this reason, numerical calculations of the ICS are now limited by the manufacturing tolerances of the trap electrode.

On the experimental side, we developed a technique to measure the ICS, completely avoiding the complex frequency shifts due to many-ion interactions. The unknown contribution of the tilt fluctuation limits our result. In the future, this uncertainty will be reduced by implementing an elaborate mechanical mechanism to adjust and cancel the tilt. In this way, the impact of tilt fluctuations, which scales linear with absolute tilt, will be significantly lowered. Second, we will use a different ion pair with very similar charge-to-mass ratio, e.g., a deuterium nucleus against a carbon nucleus. Here, the shift of the magnetron frequency difference due to tilts and ellipticity is significantly reduced. With these improvements, we will be able to measure the ICS with similar precision as our current simulations.

\section{ACKNOWLEDGMENTS}

This work was funded by the Max-Planck-Gesellschaft. J.K., S.R., and T.S. acknowledge support by the International Max Planck Research School for Precision Tests of Fundamental Symmetries (IMPRS-PTFS). F.H. acknowledges support by the International Max Planck Research School of Quantum Dynamics (IMPRS-QD). Support by the SFB 1225 (ISOQUANT) is acknowledged. T.E. acknowledges support from Academy of Finland under Project No. 295207. We thank Jiamin Hou, David B. Pinegar, Martin Höcker, Sebastian Streubel, and Christoph Diehl for earlier contributions. We thank J.V. (Trey) Porto for sharing his MATLAB code [32] with Christoph Diehl in 2008.

\section{APPENDIX A: CORRECTION OF VOLTAGE FLUCTUATIONS IN THE NEW ICS MEASUREMENT}

To reduce the statistical uncertainty of the new Ramseylike ICS measurement by one order of magnitude, we correct the measured magnetron frequencies $\left(v_{-} \propto U_{0}\right)$ of the single proton and the single ${ }^{12} \mathrm{C}^{6+}$ ion for voltage fluctuations. These voltage fluctuations are permanently monitored by measuring the axial frequency every $16 \mathrm{~min}$. Finally, the magnetron frequency is corrected for the interpolated axial frequencies $v_{z}^{\text {interpl }}$ by applying the following shift to the magnetron phase evolution time, $T_{\text {evol }}$, shown on the $x$ axis of Fig. 4: $T_{\text {evol }}^{\text {final }}=T_{\text {evol }} /\left[1-2\left(v_{z}^{\text {interpl }}-v_{z}^{\text {off }}\right) / \nu_{z}^{\text {off }}\right]$, where $\nu_{z}^{\text {off }}\left({ }^{12} \mathrm{C}^{6+}\right)=525143.659 \mathrm{~Hz}$ is a fixed offset value and $v_{z}^{\text {off }}(p)=v_{z}^{\text {off }}\left({ }^{12} \mathrm{C}^{6+}\right) / R$.

The predicted ratio of axial frequencies corrected by calculated values for trap imperfections is $R_{\text {calc }}=0.709772039(6)$. To determine this ratio, the measured axial frequency $v_{z}^{\text {off }}(p)$ and modified cyclotron frequency of the proton are corrected for (1) an energy-dependent frequency shift due to the residual magnetic inhomogeneity $B_{2}$ and (2) a tiny frequency shift, which depends on the axial dip position on the resonator, see below. After applying these corrections, we receive the 
TABLE III. Characteristics of the two axial detection systems. Here, the signal-to-noise ratio (SNR) denotes the ratio between the maximal thermal noise of the resonator and the thermal noise of the amplifier.

\begin{tabular}{lcc}
\hline \hline Properties & For ${ }^{12} \mathrm{C}^{6+}$ & For $p$ \\
\hline$v_{\text {res }}(\mathrm{Hz})$ & 525128 & 739863 \\
$Q$ value & 2500 & 4400 \\
$L(\mathrm{mH})$ & 3.4 & 1.7 \\
Dip width $(\mathrm{mHz})$ & 1100 & 660 \\
SNR $(\mathrm{dB})$ & 14 & 13 \\
\hline \hline
\end{tabular}

unshifted frequencies: $\bar{v}_{z}(p)$ and $\bar{v}_{+}(p)$. To consider also the trap-tilt, the following equation system is solved [17]:

$$
\begin{gathered}
\bar{v}_{+}^{2} \bar{v}_{z}^{2} \bar{v}_{-}^{2}=\frac{1}{4} \tilde{v}_{z}^{6}\left(1-\epsilon^{2}\right), \\
\bar{v}_{+}^{2} \bar{v}_{z}^{2}+\bar{v}_{+}^{2} \bar{v}_{-}^{2}+\bar{v}_{z}^{2} \bar{v}_{-}^{2} \\
=v_{\mathrm{c}}^{2} \tilde{v}_{z}^{2}\left(1-\frac{3}{2} \sin ^{2} \theta_{\mathrm{mag}}\right)-v_{\mathrm{c}}^{2} \tilde{v}_{z}^{2} \frac{1}{2} \epsilon \sin ^{2} \theta_{\mathrm{mag}} \\
\times \cos 2 \phi_{\mathrm{mag}}-\frac{3}{4} \tilde{v}_{z}^{4}\left(1+\frac{1}{3} \epsilon^{2}\right), \\
\bar{v}_{+}^{2}+\bar{v}_{z}^{2}+\bar{v}_{-}^{2}=v_{\mathrm{c}}^{2},
\end{gathered}
$$

where $\tilde{v}_{z}$ is the axial frequency for $\epsilon=\theta_{\text {mag }}=\phi_{\text {mag }}=0$, without any systematics. Here, $\bar{v}_{z}(p), \bar{v}_{+}(p), \epsilon$, and $\theta_{\text {mag }}$ are input parameters. By solving these equations analytically, we extract $\bar{v}_{-}(p), v_{\mathrm{c}}(p)$, and $\tilde{v}_{z}(p)$. To calculate the respective $v_{\mathrm{c}}\left({ }^{12} \mathrm{C}^{6+}\right)$ and $\tilde{v}_{z}\left({ }^{12} \mathrm{C}^{6+}\right)$ for the same $B_{0}$ and $U_{0}$, we apply the following two equations [see Eqs. (5) and (7)]:

$$
\begin{aligned}
\tilde{v}_{z}\left({ }^{12} \mathrm{C}^{6+}\right) & =\sqrt{6 \frac{m(p)}{m\left({ }^{12} \mathrm{C}^{6+}\right)}} \tilde{v}_{z}(p), \\
v_{\mathrm{c}}\left({ }^{12} \mathrm{C}^{6+}\right) & =6 \frac{m(p)}{m\left({ }^{12} \mathrm{C}^{6+}\right)} v_{\mathrm{c}}(p),
\end{aligned}
$$

where $m(p)$ and $m\left({ }^{12} \mathrm{C}^{6+}\right)$ are the masses of the proton and the carbon ion respectively [4]. Finally, the two frequency shifts due to the magnetic inhomogeneity $B_{2}$ and the dip position on the resonator have to be added on $\tilde{v}_{z}\left({ }^{12} \mathrm{C}^{6+}\right)$ to determine $\nu_{z}^{\text {off }}\left({ }^{12} \mathrm{C}^{6+}\right)$ and thus $R_{\text {calc }}$.

The second frequency correction, which we mention above, depends linearly on the detuning of the axial frequency of the ion from the resonator frequency $\nu_{\text {res }}$ of the axial detection system: $\Delta v_{z}^{\text {res }}=v_{z}^{\text {dip }}-v_{\text {res }}$; see also Table III. This small shift, which can be interpreted as an imperfect correction of the frequency pulling of the axial resonator, sometimes also denoted as the image current shift, is due to a blurring of the axial dip by voltage fluctuation during the 90 -s averaging time. Our setup allows for the first time the direct measurement of this effect by shifting the resonator by a varactor diode and measuring the axial dip for different varactor diode settings. For a ${ }^{12} \mathrm{C}^{6+}$ ion, we measured an axial frequency shift of $\delta v_{z}=-1.5(2) \times 10^{-4} \Delta v_{z}^{\text {res }}$. All systematic uncertainties of the calculated frequency ratio $R_{\text {calc }}$ are presented in Table IV.
TABLE IV. Systematic uncertainties of the calculation of the axial frequency ratio $R=v_{z}\left({ }^{12} \mathrm{C}^{6+}\right) / v_{z}(p)$. In the second column, the uncertainties of the calculated axial frequency ratio and in the third column the corresponding uncertainties of the final magnetron frequency difference are presented. A shift due to the quadratic magnetic field inhomogeneity, the dip position on the resonator, and the trap tilt had to be considered.

\begin{tabular}{lcc}
\hline \hline Effect & $\delta R_{\text {calc }}\left(10^{-9}\right)$ & $\delta\left(\Delta v_{-}\right)(\mu \mathrm{Hz})$ \\
\hline$B_{2}$ & 1.5 & 21 \\
$\theta_{\mathrm{mag}}, \phi_{\mathrm{mag}}, \epsilon$ & 5.4 & 73 \\
Dip position on the resonator & 1.0 & 13 \\
Quadratic sum & 5.7 & 77 \\
\hline \hline
\end{tabular}

Probing this prediction of the measured axial frequency ratio, 1000 measurements have been averaged, including the datasets of the ICS as well as the complete proton mass [4] measurement campaigns: $R_{\text {meas }}=0.709772038(6)$. This ratio is in excellent agreement with the calculation given above.

Further careful analysis revealed a severe limitation of such voltage correction, since by some smaller but considerable extent axial frequency fluctuations seem to be caused by fluctuations of the trap tilt. These tilt fluctuations cannot be disentangled from voltage fluctuations, but have a very different scaling than voltage fluctuations: $\delta v_{-}^{\text {tilt fluc }}(p) \approx 1.4 \times$ $10^{-5} \delta v_{z}^{\text {tilt fluc }}(p)$ and $\delta v_{-}^{\text {volt.fluc }}(p) \approx 1.3 \times 10^{-2} \delta v_{z}^{\text {volt.fluc }}(p)$.

\section{APPENDIX B: NUMERICAL UNCERTAINTY OF THE FINITE-ELEMENT SIMULATION}

To estimate the numerical uncertainty of the finite-element method, an electrode geometry should be simulated where the analytic solution is known. The relative deviation between the simulated and the analytic solution can be taken as a numerical uncertainty for various Penning traps. The simplest geometry, a hollow sphere, cannot be used as both solutions agree perfectly. An appropriate geometry is a long and closed

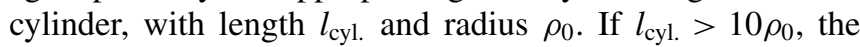
effects of the ends of the cylinder are sufficiently suppressed in the trap center and the analytical solution of an infinitely long cylinder can be used. At the position of the trapped ion, the electric field of the induced image charges $E_{\text {image }}$ is analytically described as [15,33,34]

$$
E_{\text {image }}(\rho)=1.0027 n \frac{e}{4 \pi \epsilon_{0} \rho_{0}^{3}} \rho=n \tilde{\mathcal{E}} \rho,
$$

where $\rho=|\vec{\rho}|$ is the distance of the trapped ion from the center of the cylinder. The LFG is introduced as $\tilde{\mathcal{E}}_{\rho}=$ $1.0027 \frac{e}{4 \pi \epsilon_{0} \rho_{0}^{3}}$. To model geometries with $\rho_{0}=2.7$ to $7 \mathrm{~mm}$ sufficiently well, an average mesh density of 30 to 100 mesh elements per $\mathrm{mm}^{2}$ has to be chosen. At this minimal mesh density, the result of the simulation does not change significantly, when the mesh size is decreased further. Moreover, the mesh algorithm in COMSOL MULTIPHYSICS ensures that areas with small structures, such as the slits in the electrodes, are meshed with the smallest element size. Then, the mesh size is increased gradually toward areas with less detail, e.g., the vacuum between the electrodes. Hence, the size of the largest mesh element is about $500 \mu \mathrm{m}$, whereas the smallest mesh 
TABLE $V$. The relative deviation $\Delta \mathcal{E}$ of the simulated linear field gradient $\mathcal{E}_{\rho}$ from the analytical prediction $\tilde{\mathcal{E}}_{\rho}$ [see Eq. (B1)] in a long cylinder with various radii. The optimal fit order gives the order of the point symmetric polynomial that has to be chosen to minimize $\Delta \mathcal{E}$.

\begin{tabular}{lccc}
\hline \hline Experiment & $\begin{array}{c}\text { Cylinder radius } \rho_{0} \\
(\mathrm{~mm})\end{array}$ & $\begin{array}{c}\text { Optimal fit } \\
\text { order }\end{array}$ & $\begin{array}{c}\Delta \mathcal{E} \\
\left(10^{-4}\right)\end{array}$ \\
\hline THe-Trap & 2.78 & 5 & $8.1(2.4)$ \\
LIONTRAP & 5 & 5 & $7.3(1.2)$ \\
FSU-Trap & 6.96 & 3 & $5.7(5)$ \\
\hline \hline
\end{tabular}

element is about $1 \mu \mathrm{m}$, which is ten times smaller than the manufacturing tolerances of the electrodes. One mesh element is represented by one tetrahedron.

As a consistency check, the LFG in the $z$ direction is simulated. Because of the translation invariance of an infinite cylinder, no significant LFG in this direction is expected. The resulting $\mathcal{E}_{z}=1.5(3.1) \mu \mathrm{V} / \mathrm{mm}^{2}$ is consistent with zero within the fit error bars.

The ICS and $\mathcal{E}_{\rho}$ are investigated for three different cylinder radii (see Table $\mathrm{V}$ ), corresponding to the radii of the investigated Penning-trap geometries (see Sec. VI). The relative deviation of the simulated results from the analytical prediction $\tilde{\mathcal{E}}$ is calculated as $\Delta \mathcal{E}=\left|\left(\mathcal{E}_{\rho}-\tilde{\mathcal{E}}_{\rho}\right) / \mathcal{E}_{\rho}\right|$; see Fig. 9. It turns out that higher than first orders of odd polynomials are needed to minimize $\Delta \mathcal{E}$. This is caused by the nonorthogonality of the coefficients of the polynomials. Even though only the linear order is of interest, the linear order coefficient changes if higher orders are included.

The uncertainty ( $)_{\text {num }}$ is estimated from the cylindrical test case

$$
()_{\text {num }}=\mathcal{E}_{\rho, z} \Delta \mathcal{E}
$$

where $\mathcal{E}_{\rho, z}$ are the LFG of the simulated trap geometries and $\Delta \mathcal{E}$ is taken from Table $\mathrm{V}$.

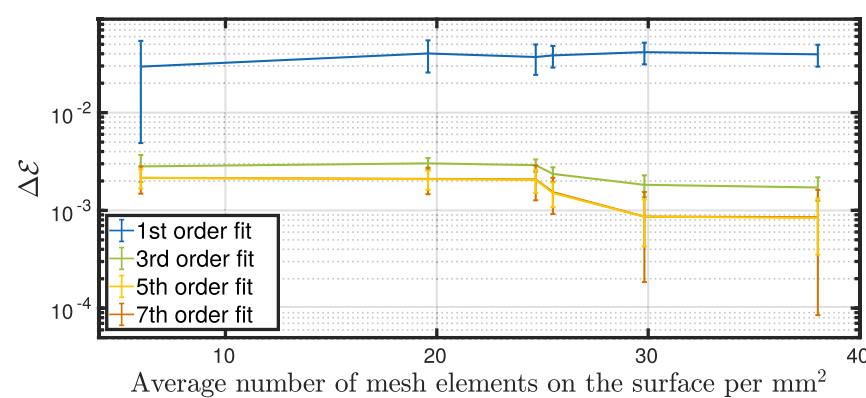

FIG. 9. Relative deviation of the simulated ICS of a pointlike charge inside a hollow cylinder with radius $2.78 \mathrm{~mm}$ and a total length of $30 \mathrm{~mm}$ compared to the analytical calculation as a function of the mesh size. While the linear fit does not show any significant decrease of $\Delta \mathcal{E}$ for a finer mesh, higher order polynomials do. Finally, the fifth-order polynomial is sufficient to minimize $\Delta \mathcal{E}$. Higher order polynomials than the fifth order describe the data as well as the fifth-order polynomial but with a larger uncertainty.

\section{APPENDIX C: SEMIANALYTICAL TREATMENT}

This section recapitulates Porto's method [14], focusing entirely on image charges, while following Porto's notation closely. ${ }^{5}$ This method can be also used to calculate components of the electrostatic potential when voltages are applied to the electrodes. Let us consider a pointlike ion with charge $q$ at position $\boldsymbol{r}^{\prime}$ in the trap with all electrodes grounded. The total potential at the general position $\boldsymbol{r}$ is then given by

$$
\begin{aligned}
\Phi_{\text {ion }}\left(\boldsymbol{r}, \boldsymbol{r}^{\prime}\right) & =\frac{q}{4 \pi \varepsilon_{0}} G\left(\boldsymbol{r}, \boldsymbol{r}^{\prime}\right) \\
& =\frac{q}{4 \pi \varepsilon_{0}}\left[\frac{1}{\left|\boldsymbol{r}-\boldsymbol{r}^{\prime}\right|}+F\left(\boldsymbol{r}, \boldsymbol{r}^{\prime}\right)\right],
\end{aligned}
$$

where the first term describes the ion's Coulomb potential and the second term is due to the image charges. The implicitly defined $G\left(\boldsymbol{r}, \boldsymbol{r}^{\prime}\right)$ is the spatially dependent potential. The resulting electric field at the position of the ion is then calculated as

$$
\boldsymbol{E}_{\text {image }}\left(\boldsymbol{r}^{\prime}\right)=-\left.\frac{q}{4 \pi \varepsilon_{0}} \nabla_{\boldsymbol{r}} F\left(\boldsymbol{r}, \boldsymbol{r}^{\prime}\right)\right|_{\boldsymbol{r}=\boldsymbol{r}^{\prime}} .
$$

First, the expression is derived with respect to the general coordinate $\boldsymbol{r}$. Then, the substitution $\boldsymbol{r} \rightarrow \boldsymbol{r}^{\prime}$ is made in order to evaluate the electric field at the position $\boldsymbol{r}^{\prime}$ of the ion.

We now turn to the condition that determines the contribution $F\left(\boldsymbol{r}, \boldsymbol{r}^{\prime}\right)$ of the image charges to the total potential. Let $\boldsymbol{\Omega}$ denote the conducting trap surfaces, which are held at ground. Since these (perfectly) conducting surfaces remain equipotential surfaces even in the presence of the ion with its Coulomb field, the total potential on the trap surfaces must vanish: $\Phi_{\text {ion }}\left(\boldsymbol{\Omega}, \boldsymbol{r}^{\prime}\right)=0$. The contribution by the image charges must therefore compensate for the ion's Coulomb potential. Like any source-free electrostatic potential, the potential produced by the image charges fulfils the Laplace equation $\triangle_{r} F\left(\boldsymbol{r}, \boldsymbol{r}^{\prime}\right)=$ 0 inside the trap, where there are no image charges. The most general solution without singularities is given in terms of associated Legendre polynomials $P_{l}^{m}$ by

$$
\begin{aligned}
F\left(\boldsymbol{r}, \boldsymbol{r}^{\prime}\right)= & \frac{1}{d} \sum_{l=0}^{\infty}\left(\frac{r}{d}\right)^{l} \sum_{m=0}^{l} P_{l}^{m}(\cos \theta) \\
& \times\left[C_{l}^{m}\left(\boldsymbol{r}^{\prime}\right) \cos (m \phi)+S_{l}^{m}\left(\boldsymbol{r}^{\prime}\right) \sin (m \phi)\right],
\end{aligned}
$$

where $C_{l}^{m}\left(\boldsymbol{r}^{\prime}\right)$ and $S_{l}^{m}\left(\boldsymbol{r}^{\prime}\right)$ are dimensionless coefficients. These depend on the position $\boldsymbol{r}^{\prime}$ of the ion. To save some space by not having to distinguish between the cases of $m=0$ and $m \neq 0$, we use the convention $P_{l}^{0} \equiv P_{l}$, where $P_{l}$ is the ordinary Legendre polynomial. As usual, we use $r=|\boldsymbol{r}|$ as the length of the vector $\boldsymbol{r}$, and we will employ this convention with the symbol $r^{\prime}$ to the position $\boldsymbol{r}^{\prime}$ of the ion shortly. The characteristic trap dimension $d$ from Eq. (2) has been included in the denominator in order to emphasize that the overall term has the dimension of inverse length, like the $1 /\left|\boldsymbol{r}-\boldsymbol{r}^{\prime}\right|$ term

\footnotetext{
${ }^{5}$ If you are familiar with Porto's paper [14], brace for four minor differences here: (1) the use of the constant $1 /\left(4 \pi \varepsilon_{0}\right)$ in conjunction with the charge for SI units; (2) no separate expressions for $m=0$ and $m>0$; (3) no use of the truncation parameter $N$ as an index; and (4) no suppression of the superscript $m$.
} 
in the Coulomb potential. For $r^{\prime}<r$, this term is expressed in products of associated Legendre polynomials as

$$
\begin{aligned}
\frac{1}{\left|\boldsymbol{r}-\boldsymbol{r}^{\prime}\right|}= & \frac{1}{r} \sum_{l=0}^{\infty}\left(\frac{r^{\prime}}{r}\right)^{l} \sum_{m=0}^{l} \epsilon_{l}^{m} \\
& \times P_{l}^{m}(\cos \theta) P_{l}^{m}\left(\cos \theta^{\prime}\right) \cos \left[m\left(\phi-\phi^{\prime}\right)\right]
\end{aligned}
$$

with the factor

$$
\epsilon_{l}^{m}=\left\{\begin{array}{ll}
1 & \text { for } m=0 \\
2 \frac{(l-m) !}{(l+m) !} & \text { for } m \neq 0
\end{array} .\right.
$$

With the trigonometric angle sum identity

$$
\begin{aligned}
\cos \left[m\left(\phi-\phi^{\prime}\right)\right]= & \cos (m \phi) \cos \left(m \phi^{\prime}\right) \\
& +\sin (m \phi) \sin \left(m \phi^{\prime}\right),
\end{aligned}
$$

the similarity between the expansions (C4) and (C5) is enhanced.

For technical reasons, we truncate the infinite series at a maximum $l_{\max }=N$. While this probably means that the approximate potential $\Phi_{\text {ion }}\left(\boldsymbol{r}, \boldsymbol{r}^{\prime}\right)$, or equivalently $G\left(\boldsymbol{r}, \boldsymbol{r}^{\prime}\right)$, no longer vanishes exactly on the trap electrodes, we check how well the condition $G\left(\boldsymbol{\Omega}, \boldsymbol{r}^{\prime}\right)=0$ is met by integrating the squared deviation $\left[G\left(\boldsymbol{r}, \boldsymbol{r}^{\prime}\right)\right]^{2}$ over the trap surfaces $\boldsymbol{\Omega}$. The goal is to choose the coefficients $C_{l}^{m}\left(\boldsymbol{r}^{\prime}\right)$ and $S_{l}^{m}\left(\boldsymbol{r}^{\prime}\right)$ such that the expression

$$
\int_{\Omega}\left[G\left(\boldsymbol{r}, \boldsymbol{r}^{\prime}\right)\right]^{2} d \Omega
$$

is minimized.

Plugging in the expansions (C4) and (C5) and carrying out the square result in trigonometric cross terms of the kinds $\cos \left(m_{1} \phi\right) \cos \left(m_{2} \phi\right)$ and $\sin \left(m_{1} \phi\right) \sin \left(m_{2} \phi\right)$. Upon integration over $\phi$ from 0 to $2 \pi$, these terms vanish for two integers with $m_{1} \neq m_{2}$. For this integration range, the cross terms $\cos \left(m_{1} \phi\right) \sin \left(m_{2} \phi\right)$ vanish for arbitrary integers $m_{1}$ and $m_{2}$. This reminds us of the fact that the cosine and the sine terms in the solution (C4) of the Laplace equation represent two independent degrees of freedom. The cancellation of different $m$ cross terms for cylindrical symmetry means that the contributions of terms with $\cos (m \phi)$ and $\sin (m \phi)$ can be minimized separately. This tremendous simplification is no longer applicable without cylindrical symmetry, thereby greatly complicating the matter.

In Ref. [15], the effect of the slits-both vertical and horizontal-is estimated by calculating the image charge distribution initially without the slits and then ignoring the image charges that sit at the position of the slits, assuming that the slits do not lead to a major redistribution of image charges elsewhere on the remaining electrode surfaces. Along these lines, reducing the integration range of the angle variable $\phi$ might have constituted a first attempt to account for the effect of the vertical slit here. However, we have discussed above that such a reduction has unintended consequences.

Having convinced ourselves of the importance of cylindrical symmetry for the method and shied away from the computational complexities that result in its absence, we continue on the same path as Ref. [14]. ${ }^{6}$ With cylindrical symmetry, we are free to place the ion on the $x$ axis without loss of generality. Because of the choice of $\phi^{\prime}=0$, the sine terms $\sin (m \phi)$ vanish in the expansion (C5), necessitating $S_{l}^{m}\left(\boldsymbol{r}^{\prime}\right)=0$ to remove the sine term in Eq. (C4). We are thus left with the cosine terms $\cos (m \phi)$ and the optimization of the coefficients $C_{l}^{m}\left(\boldsymbol{r}^{\prime}\right)$. For a fixed $m$, the expression that needs to be minimized reads

$$
\begin{aligned}
& \int_{\Omega}\left\{\sum _ { l = m } ^ { N } \left[\frac{1}{d}\left(\frac{r}{d}\right)^{l} P_{l}^{m}(\cos \theta) C_{l}^{m}\left(\boldsymbol{r}^{\prime}\right)\right.\right. \\
& \left.\left.+\frac{\epsilon_{l}^{m}}{r}\left(\frac{r^{\prime}}{r}\right)^{l} P_{l}^{m}(\cos \theta) P_{l}^{m}\left(\cos \theta^{\prime}\right)\right]\right\}^{2} d \Omega .
\end{aligned}
$$

Deriving this expression with respect to the specific coefficient $C_{l}^{m}\left(\boldsymbol{r}^{\prime}\right)$ and equating the result to zero yields the equation

$$
\sum_{l=m}^{N} B_{k l}^{m} C_{l}^{m}\left(\boldsymbol{r}^{\prime}\right)=-\sum_{l=m}^{N} D_{k l}^{m} A_{l}^{m}\left(\boldsymbol{r}^{\prime}\right)
$$

with

$$
\begin{gathered}
A_{l}^{m}\left(\boldsymbol{r}^{\prime}\right)=\left(\frac{r^{\prime}}{d}\right)^{l} P_{l}^{m}\left(\cos \theta^{\prime}\right), \\
B_{k l}^{m}=\int_{\Omega}\left(\frac{r}{d}\right)^{k+l} P_{k}^{m}(\cos \theta) P_{l}^{m}(\cos \theta) d \Omega, \\
D_{k l}^{m}=\epsilon_{l}^{m} \int_{\Omega}\left(\frac{r}{d}\right)^{k-l-1} P_{k}^{m}(\cos \theta) P_{l}^{m}(\cos \theta) d \Omega .
\end{gathered}
$$

In this process, the order of the integration and the summation were exchanged, which is fine because the sum involves only a finite number of terms after truncation. Equation (C10) still involves a sum over the coefficients $C_{l}^{m}\left(\boldsymbol{r}^{\prime}\right)$. Formally, the solution for a single coefficient is given by

$$
C_{l}^{m}\left(\boldsymbol{r}^{\prime}\right)=\sum_{k=m}^{N} C_{l k}^{m} A_{k}^{m}\left(\boldsymbol{r}^{\prime}\right)
$$

with the element $C_{l k}^{m}=-\left[\left(B^{m}\right)^{-1} D^{m}\right]_{l k}$. The missing subscripts of $B^{m}$ and $D^{m}$ indicate matrices with entries $B_{k l}^{m}$ and $D_{k l}^{m}$ according to Eqs. (C12) and (C13), respectively. The superscript ${ }^{-1}$ stands for the inverse matrix.

In combination with Eq. (C11), it becomes clear that the coefficient $C_{l}^{m}\left(\boldsymbol{r}^{\prime}\right)$ contains a sum over terms $\left(r^{\prime}\right)^{k} P_{k}^{m}\left(\cos \theta^{\prime}\right)$. These are multiplied with similar terms of the kind $r^{l} P_{l}^{m}(\cos \theta)$ in the image term (C4). We now need to select the right combination of $k, l$, and $m$ that produce the relevant lowest-order terms. The general conversion

$$
\begin{aligned}
r^{l} P_{l}^{m}(\cos \theta)= & (-1)^{m} \frac{(l+m) !}{2^{m}} \\
& \times \sum_{i=0}^{\left\lfloor\frac{l-m}{2}\right\rfloor} \frac{(-1)^{i}}{2^{2 i}} \frac{z^{l-m-2 i} \rho^{m+2 i}}{i !(m+i) !(l-m-2 i) !}
\end{aligned}
$$

\footnotetext{
${ }^{6}$ Remarkably, Ref. [14] considers boundary conditions without cylindrical symmetry in its Eq. (13) when voltages are applied to the electrodes.
} 
TABLE VI. Conversion of $r^{l} P_{l}^{m}(\cos \theta)$ from spherical to cylindrical coordinates for the lowest-order terms.

\begin{tabular}{lccc}
\hline \hline$r^{l} P_{l}^{m}(\cos \theta)$ & $m=0$ & $m=1$ & $m=2$ \\
\hline$l=0$ & 1 & 0 & 0 \\
$l=1$ & $z$ & $-\rho$ & 0 \\
$l=2$ & $z^{2}-\frac{\rho^{2}}{2}$ & $-3 z \rho$ & $3 \rho^{2}$ \\
\hline \hline
\end{tabular}

from spherical to cylindrical coordinates [23] is helpful by showing that the resulting polynomial in $z$ and $\rho$ is homogeneous with degree $l$.

Since we are interested in terms that are linear in either $\rho^{\prime}$ or $z^{\prime}$ after taking a derivative with respect to $\rho$ or $z$ and then making the substitution $\rho \rightarrow \rho^{\prime}$ or $z \rightarrow z^{\prime}$ in order to evaluate the term at the position of the ion, we do not need to look beyond degree 2 . These first few terms are shown in Table VI.

Terms without dependence on $\rho$ or $z$, respectively, can be ignored right away. Consequently, the expressions must at least be of degree 1 in $\rho$ or $z$. The lowest-order terms that contribute to the image-charge effect are given by

$$
F\left(\boldsymbol{r}, \boldsymbol{r}^{\prime}\right) \simeq \frac{1}{d^{3}}\left[C_{11}^{0} z^{\prime} z+C_{11}^{1} \rho^{\prime} \rho+C_{20}^{0}\left(z^{2}-\frac{\rho^{2}}{2}\right)\right]
$$

with the coefficients from Eq. (C14). Following the procedure from Eq. (C3) and comparing it with Eq. (9) yields the linear field gradients

$$
\begin{aligned}
& \mathcal{E}_{\rho}=-\frac{e}{4 \pi \varepsilon_{0} d^{3}}\left(C_{11}^{1}-C_{20}^{0}\right), \\
& \mathcal{E}_{z}=-\frac{e}{4 \pi \varepsilon_{0} d^{3}}\left(C_{11}^{0}+2 C_{20}^{0}\right) .
\end{aligned}
$$

The shift (14) to the free-space cyclotron frequency via the invariance theorem is rewritten as

$$
\Delta v_{\mathrm{c}} \approx-\frac{n e}{16 \pi^{2} \varepsilon_{0} d^{3} B_{0}}\left(2 C_{11}^{1}+C_{11}^{0}\right)
$$

It does not depend on the coefficient $C_{20}^{0}$. This was already to be expected from the spatial shape of the associated potential (C16), which is the same as the ideal quadrupole potential (1) in the trap. Consequently, the coefficient $C_{20}^{0}$ effectively describes a slight modification of the trapping potential, which is covered by the invariance theorem (8).

Having established the theoretical foundations of the method, we turn to some practical simplifications for the traps that we will be modeling. These models are not only cylindrically symmetric, they possess mirror symmetry at the $x y$ plane, too. Since the parity of the associated Legendre polynomials $P_{l}^{m}$ flips for different parities of $l$, the integrals $(\mathrm{C} 12)$ and $(\mathrm{C} 13)$ for the matrix elements $B_{k l}^{m}$ and $D_{k l}^{m}$, respectively, vanish over a trap with such mirror symmetry when $k$ and $l$ have opposite parity. Thus, we limit ourselves to the calculation of submatrices with equal parity of $k$ and $l$-either both even or both odd - and we reduce the integration to the upper octants (and the $x y$ plane), where $0 \leqslant \cos (\theta) \leqslant 1$ or, equivalently, $z \geqslant 0$. Such a quadratic submatrix has a sizenumber of rows or columns-of

$$
N_{\mathrm{sp}}=\left\lceil\frac{N-m+1}{2}\right\rceil
$$

when $m$ has the same parity as $k$ and $l$ and a size of

$$
N_{\text {op }}=\left\lfloor\frac{N-m+1}{2}\right\rfloor
$$

when $m$ has the opposite parity of $k$ and $l$. The subscripts "sp" and "op" are short for same parity and opposite parity, respectively. We will use $N_{\text {sm }}$ for the size of either submatrix in contrast to the size $N$. Roughly speaking, the submatrices that we deal with have size $N / 2$, give or take 0.5 to 1 .

Because of cylindrical symmetry, the integration over the surfaces reduces to a one-dimensional integration with a surface factor. With the trap surfaces described as functions $\rho(z)$ or $z(\rho)$ that are revolved around the $z$ axis, the corresponding surface factors to replace $d \Omega$ are given by

$$
2 \pi \rho(z) \sqrt{1+\left(\frac{\partial \rho}{\partial z}\right)^{2}} d z \quad \text { and } \quad 2 \pi \rho \sqrt{1+\left(\frac{\partial z}{\partial \rho}\right)^{2}} d \rho
$$

respectively. For most geometries, the square roots in the surface factors shatter all hopes of analytical integration. That is why Porto's method is referred to as semianalytical: The integration and the inversion of the matrices require numerical methods. Combined with the mirror symmetry, the description of the trap surfaces as surfaces of revolution explains why the traps are defined entirely by their cross section in the first quadrant; see Fig. 8.

Before we finally get to results of the actual calculation, we pick up on one of Porto's suggestions - a weighting function $w(\boldsymbol{r})$. Instead of minimizing $\left[G\left(\boldsymbol{r}, \boldsymbol{r}^{\prime}\right)\right]^{2}$ as in Eq. (C8), one can minimize $\left[w(\boldsymbol{r}) G\left(\boldsymbol{r}, \boldsymbol{r}^{\prime}\right)\right]^{2}$ over the trap surfaces because $G\left(\boldsymbol{r}, \boldsymbol{r}^{\prime}\right)$ is supposed to vanish there. Porto suggested using $1 / r^{3}$ as a weighting function [14] in order to relax "the boundary condition on distant parts of the trap in such a way that errors in the approximate solution of $G\left(\boldsymbol{r}, \boldsymbol{r}^{\prime}\right)$ contribute equally from all parts of the trap" (p. 023403-4). In fact, any weighting of the kind $r^{w}$, where $w$ is a number rather than a function here is readily implemented by including $w$ in the exponent of $r$ in the integrals (C12) and (C13).

We have worked with $w=0$ (no weight) and $w=-3$ to check whether the solutions converge to the same value. If they do within the error that we attribute to each solution individually, we feel confident saying that the solutions have converged accurately. Otherwise, we would have checked for numerical issues or assigned a systematic uncertainty. In general, the coefficients calculated with the weight $w=-3$ are initially closer to the limiting value at a given size of the submatrix, but the plateau is reached at similar sizes for both weights (see Fig. 10).

While Porto's 2001 publication [14] truncated the series at $N=44$, thereby using associated Legendre polynomials $P_{l}^{m}$ up to degree $N$, it was possible to go up to about $N=480$ using MATLAB. However, there is little use in pushing $N$ to the numerical limits because the scatter of the solution increases well before due to numerical instabilities. Fortunately, the results agree apart from occasional outliers. A reasonable choice that almost certainly contains the region of greatest stability is $N=200$. Problems with the numerical integration, 

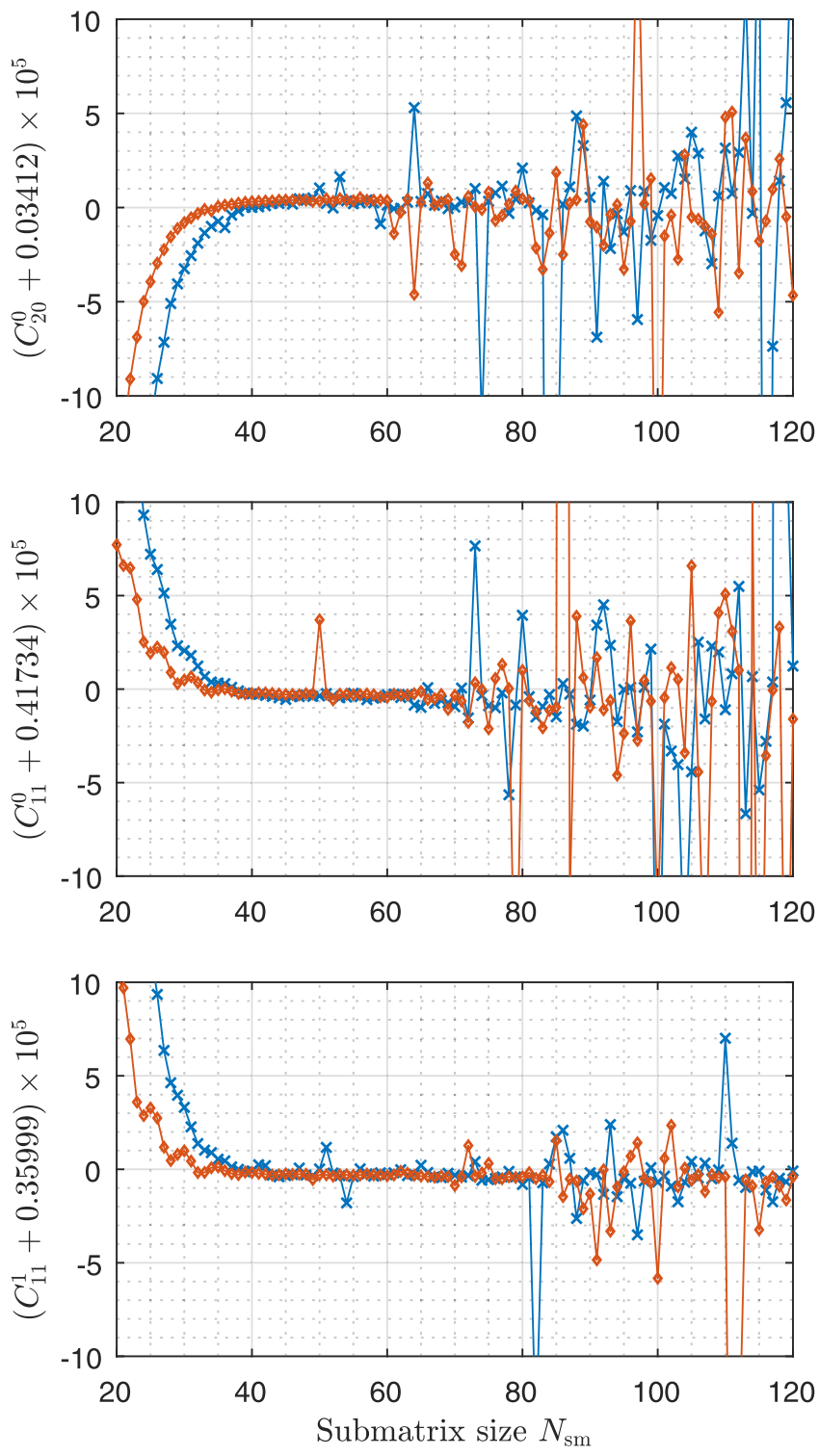

FIG. 10. The evolution of the coefficients for the Porto-Trap as a function of submatrix size. The values from Table VII have been subtracted and the difference scaled by $10^{5}$ such that one unit on the vertical axis corresponds to a change of 1 in the least significant digit of the quoted coefficients. The blue crosses and red open diamonds correspond to a weight of $w=0$ and $w=-3$, respectively. The lines connecting the points serve as a guide to the eye in terms of order and highlight data outside the selected range.

which are particularly prevalent at larger $m,^{7}$ slow down the evaluation disproportionately. The offending part of the integrand is often found near $\cos \theta=1$, that is, on or close to the $z$ axis. Here, certain combinations of the associated Legendre polynomials $P_{k}^{m} P_{l}^{m}$ in the integrals (C12) and (C13) become steeply oscillatory and thus notoriously difficult to

\footnotetext{
${ }^{7}$ Here, large $m$ starts at 1 . In order to calculate higher order contributions to the image-charge potential, which are not featured in this article, matrices up to $m=3$ were calculated, and the problem was found to become even more prevalent.
}

evaluate. As the matrix elements (C12) and (C13) do not depend on $N$-apart from the fact that they are not calculated for $k>N$ or $l>N$-one always has the option of using fewer matrix elements than one has calculated. In fact, this is how the convergence is checked.

\section{Porto-Trap}

Since the coefficients $C_{20}^{0}=0.0629(5), C_{11}^{0}=0.4174(3)$, and $C_{11}^{1}=0.3603$ (4) for Porto's model of the FSU-Trap are shown in his original publication [14], this trap became the first test case. The two characteristic dimensions are $z_{0}=$ $6.00 \mathrm{~mm}$ and $\rho_{0}=6.96 \mathrm{~mm}$. For the semianalytical calculation, the trap is truncated at a distance of $2.2 d$, and the remaining openings are closed by spherical segments, sloppily called backplane despite the spherical shape. Such backplanes which are not actual trap surfaces may be necessary for numerical stability. When too large a range of angle $\theta$ is left unconstrained by not subtending any electrode, the minimization may try to push most of the overlap into the gap, thereby leading to a bad solution on the trap electrodes.

Despite the truncating backplane being artificial, one has to resist the urge of retracting it as far as possible because the integrals (C12) and (C13) depend strongly on the distance $r$ from the origin, most notably for large $k$ and $l$ in the case of $B_{k l}^{m}$ and a strong difference between $k$ and $l$ in the case of $D_{k l}^{m}$. If the distances $r$ vary considerably over the trap electrodes that are modeled, the numerical precision may become insufficient as the contributions to the overlap from the nearby surfaces, which dominate the image charge effect, are swamped by larger contributions from the most distant surfaces, which play a much smaller role in terms of image charges. We will face this problem to a larger extent when dealing with a cylindrical trap in Sec. C3. Hyperboloidal traps are much easier to calculate than open-end-cap ${ }^{8}$ cylindrical traps [36] in this regard because the ring and the end cap are at comparable distances, resulting in much less of a variation in $r$ over these electrodes.

Figure 10 shows the evolution of the coefficients for the Porto-Trap. At a submatrix size of about $N_{\mathrm{sm}}=40$-above the submatrix sizes of 22 or 23 that result from the maximum $N=44$ of Ref. [14] according to Equations (C20) and (C21) - the trend in the coefficients for Porto's trap flattens out to better than $10^{-5}$ on an absolute scale. At about $N_{\mathrm{sm}}=$ 60 , the scatter increases visibly. Yet, the overwhelming majority of the points still deviates by less than $10^{-4}$ up to $N_{\mathrm{sm}}=$ 100. We attribute the scatter to increased numerical instability rather than poor convergence of the solution because there is no general trend in the coefficients. Averaging over the range of least scatter from 40 to 60 gives the values shown in Table VII. The standard deviation in this region of largest stability-and we hope the most accurate convergence-is even smaller than the error we give. Rather than go for another digit, we tend to be a bit more cautious. We inspect medians,

\footnotetext{
${ }^{8}$ In terms of distances, cylindrical traps with flat-plate end caps [35] are much more similar to hyperboloidal traps, and they are closed almost naturally. Consequently, Porto used such a trap to verify his calculation of the electrostatic potential [14].
} 
TABLE VII. Low-order coefficients of the image-charge potential (C16) for different traps as outcome of the semianalytical approach introduced above. These coefficients are needed to calculate the ICS, based on (C19).

\begin{tabular}{lccc}
\hline \hline & $C_{20}^{0}$ & $C_{11}^{0}$ & $C_{11}^{1}$ \\
\hline Porto-Trap & $-0.03412(1)$ & $-0.41734(1)$ & $-0.35999(1)$ \\
THe-Trap & $-0.05419(8)$ & $-0.42945(3)$ & $-0.33683(3)$ \\
LIONTRAP & $+0.21945(5)$ & $-0.43890(10)$ & $-0.84928(10)$ \\
\hline \hline
\end{tabular}

which are less sensitive to outliers than averages, and play with the ranges in order to ensure that such alternate values are within the range of errors we quote. The results obtained with our code for all four traps are summarized in Table VII.

\section{THe-Trap}

The geometry is based on the trap shown in Fig. 8. First, the end cap is extended all the way to the $z$ axis, thereby closing the hole. Then, the electrodes are truncated and closed by a sphere with a radius of $2.5 d$. This truncation radius is larger than the $2.2 d_{\mathrm{FSU}}$ in Porto's model of the FSU-Trap because the tip of the guard electrode in THe-Trap is at a distance of $2.22 d$. In order to retain at least some properties of the guard electrode, the truncation radius had be to increased.

Because of the anisotropic shrinking of the THe-Trap geometry and the following displacement of the end cap, THe-Trap needs a slightly more complicated routine for the integration over the end cap than the two previous hyperboloidal traps.

The coefficients whose averages are shown in Table VII are most stable between submatrix sizes of 45 and 65 . The overall scatter is larger than for the previous trap, which is why larger errors have been assigned.

\section{LIONTRAP}

The mathematical model of this cylindrical trap is based on Fig. 8. All the slits are closed on the inside, and a spherical cap is put on the remaining opening. Overall, there are four distinct surfaces. The characteristic trap dimension $d$ shown in Table II results from Eq. (2) with $\rho_{0}=5.00 \mathrm{~mm}$ (the inner radius for most of the trap, most notably near the center) and $z_{0}=6.8220 \mathrm{~mm}$ (the smallest axial coordinate of the end caps).

The most stable range for the coefficients lies between submatrix sizes of 15 to 35 . For larger values, the coefficients scatter on an absolute level of $10^{-3}$, which is the largest scatter of all traps considered here. We attribute the issue to numerical problems that arise from the distance having to cover the largest range. The dimensionless distance $r / d$ reaches from 0.92 at the electrode closest to the center to 3.70 at the furthest electrode, thus varying by a factor of 4 , which is almost twice the factor for the hyperboloidal trap. A similar argument explains why this trap produces the largest deviation between the solutions for the two different weights $w=0$ and $w=-3$.

We test the result for the radial gradient against the analytical prediction [see Eq. (B1)] for a cylinder of infinite length and inner radius $\rho_{0}$. Comparing it with Eq. (C17) shows that, in this case, the difference of two coefficients would yield the value

$$
C_{11}^{1}-C_{20}^{0}=-1.0027\left(\frac{d}{\rho_{0}}\right)^{3},
$$

which is -1.06871 when plugging in values on the right-hand side and $-1.06873(12)$ when plugging in the values on the left-hand side. When ignoring the slits, the LIONTRAP seems to be reasonably close to an infinite cylinder as far as imagecharge fields are concerned. We can interpret the agreement as a success of Porto's semianalytical method. On the other hand, the effort may not be warranted if there is so little to be gained. Unfortunately, a more accurate representation of reality requires a method that manages to deal with the slits in the electrodes.
[1] S. Rainville, J. K. Thompson, and D. E. Pritchard, Science 303, 334 (2004).

[2] S. L. Zafonte and R. S. Van Dyck Jr., Metrologia 52, 280 (2015).

[3] E. G. Myers, A. Wagner, H. Kracke, and B. A. Wesson, Phys. Rev. Lett. 114, 013003 (2015).

[4] F. Heiße, F. Köhler-Langes, S. Rau, J. Hou, S. Junck, A. Kracke, A. Mooser, W. Quint, S. Ulmer, G. Werth, K. Blaum, and S. Sturm, Phys. Rev. Lett. 119, 033001 (2017).

[5] S. Sturm, G. Werth, and K. Blaum, Ann. Phys. 525, 620 (2013).

[6] A. Wagner, S. Sturm, F. Köhler, D. A. Glazov, A. V. Volotka, G. Plunien, W. Quint, G. Werth, V. M. Shabaev, and K. Blaum, Phys. Rev. Lett. 110, 033003 (2013).

[7] F. Köhler, K. Blaum, M. Block, S. Chenmarev, S. Eliseev, D. A. Glazov, M. Goncharov, J. Hou, A. Kracke, D. A. Nesterenko, Y. N. Novikov, W. Quint, E. M. Ramirez, V. M. Shabaev, S. Sturm, A. V. Volotka, and G. Werth, Nat. Commun. 7, 10246 (2016).
[8] H. Nagahama, C. Smorra, S. Sellner, J. Harrington, T. Higuchi, M. J. Borchert, T. Tanaka, M. Besirli, A. Mooser, G. Schneider, K. Blaum, Y. Matsuda, C. Ospelkaus, W. Quint, J. Walz, Y. Yamazaki, and S. Ulmer, Nat. Commun. 8, 14084 (2017)

[9] G. Schneider, A. Mooser, M. Bohman, N. Schön, J. Harrington, T. Higuchi, H. Nagahama, S. Sellner, C. Smorra, K. Blaum, Y. Matsuda, W. Quint, J. Walz, and S. Ulmer, Science 358, 1081 (2017).

[10] J. Thompson, E. Myers, J. Brown, M. Dewey, and S. Rainville, Nature (London) 438, 1096 (2005).

[11] S. Sturm, F. Köhler, J. Zatorski, A. Wagner, Z. Harman, G. Werth, W. Quint, C. H. Keitel, and K. Blaum, Nature (London) 506, 467 (2014).

[12] R. S. Van Dyck Jr., D. B. Pinegar, S. Van Liew, and S. L. Zafonte, Int. J. Mass Spectrom. 251, 231 (2006). 
[13] R. S. Van Dyck Jr., F. L. Moore, D. L. Farnham, and P. B. Schwinberg, Phys. Rev. A 40, 6308 (1989).

[14] J. V. Porto, Phys. Rev. A 64, 023403 (2001).

[15] S. Sturm, A. Wagner, M. Kretzschmar, W. Quint, G. Werth, and K. Blaum, Phys. Rev. A 87, 030501(R) (2013).

[16] N. F. Ramsey, Phys. Rev. 76, 996 (1949).

[17] L. S. Brown and G. Gabrielse, Rev. Mod. Phys. 58, 233 (1986).

[18] K. Blaum, Phys. Rep. 425, 1 (2006).

[19] D. Wineland and H. Dehmelt, J. Appl. Phys. 46, 919 (1975).

[20] G. Gabrielse, Phys. Rev. A 29, 462 (1984).

[21] D. F. A. Winters, M. Vogel, D. M. Segal, and R. C. Thompson, J. Phys. B 39, 3131 (2006).

[22] M. Kretzschmar, Appl. Phys. B 107, 1007 (2012).

[23] J. Ketter, Theoretical treatment of miscellaneous frequencyshifts in Penning traps with classical perturbation theory, Ph.D. thesis, Ruprecht-Karls-Universität, Heidelberg, Germany, 2015.

[24] H. Häffner, Präzisionsmessung des magnetischen Moments des Elektrons in wasserstoffähnlichem Kohlenstoff, Ph.D. thesis, Universität Mainz, Mainz, Germany, 2000 (unpublished).

[25] E. A. Cornell, R. M. Weisskoff, K. R. Boyce, and D. E. Pritchard, Phys. Rev. A 41, 312 (1990).
[26] J. L. Verdú, S. Djekić, S. Stahl, T. Valenzuela, M. Vogel, G. Werth, H.-J. Kluge, and W. Quint, Phys. Scr. 2004, 68 (2004).

[27] S. Sturm, A. Wagner, B. Schabinger, and K. Blaum, Phys. Rev. Lett. 107, 143003 (2011).

[28] G. Gabrielse, Phys. Rev. Lett. 102, 172501 (2009).

[29] Comsol Multiphysics, version 5.2 (2017), www. comsol.com.

[30] S. Sturm, A. Wagner, B. Schabinger, J. Zatorski, Z. Harman, W. Quint, G. Werth, C. H. Keitel, and K. Blaum, Phys. Rev. Lett. 107, 023002 (2011).

[31] F. Köhler, Bound-electron $g$-factor measurements for the determination of the electron mass and isotope shifts in highly charged ions, Ph.D. thesis, Ruprecht-Karls-Universität, Heidelberg, Germany, 2015.

[32] J. V. Porto (private communication).

[33] M. D. Tinkle and S. E. Barlow, J. Appl. Phys. 90, 1612 (2001).

[34] S. E. Barlow, J. Appl. Phys. 94, 6221 (2003).

[35] G. Gabrielse and F. C. Mackintosh, Int. J. Mass Spectrom. Ion Processes 57, 1 (1984)

[36] G. Gabrielse, L. Haarsma, and S. L. Rolston, Int. J. Mass Spectrom. Ion Processes 88, 319 (1989). 